TRANSACTIONS OF THE

AMERICAN MATHEMATICAL SOCIETY

Volume 358, Number 5 , Pages 1983-2010

S 0002-9947(05)03986-3

Article electronically published on December 20, 2005

\title{
FILTRATIONS IN SEMISIMPLE LIE ALGEBRAS, I
}

\author{
Y. BARNEA AND D. S. PASSMAN
}

\begin{abstract}
In this paper, we study the maximal bounded $\mathbb{Z}$-filtrations of a complex semisimple Lie algebra $L$. Specifically, we show that if $L$ is simple of classical type $A_{n}, B_{n}, C_{n}$ or $D_{n}$, then these filtrations correspond uniquely to a precise set of linear functionals on its root space. We obtain partial, but not definitive, results in this direction for the remaining exceptional algebras. Maximal bounded filtrations were first introduced in the context of classifying the maximal graded subalgebras of affine Kac-Moody algebras, and the maximal graded subalgebras of loop toroidal Lie algebras. Indeed, our main results complete this classification in most cases. Finally, we briefly discuss the analogous question for bounded filtrations with respect to other Archimedean ordered groups.
\end{abstract}

\section{INTRODUCTION}

Let $L$ be a Lie algebra over a field $K$. A $\mathbb{Z}$-filtration $\mathcal{F}=\left\{F_{i} \mid i \in \mathbb{Z}\right\}$ of $L$ is a collection of $K$-subspaces

$$
\cdots \subseteq F_{-2} \subseteq F_{-1} \subseteq F_{0} \subseteq F_{1} \subseteq F_{2} \subseteq \cdots
$$

indexed by the integers $\mathbb{Z}$ such that $\left[F_{i}, F_{j}\right] \subseteq F_{i+j}$ for all $i, j \in \mathbb{Z}$. One usually also assumes that $\bigcup_{i} F_{i}=L$ and $\bigcap_{i} F_{i}=0$. In particular, $F_{0}$ is a Lie subalgebra of $L$ and each $F_{i}$ is an $F_{0}$-Lie submodule of $L$. Furthermore, we say that the filtration is bounded if there exist integers $\ell$ and $\ell^{\prime}$ with $F_{\ell}=0$ and $F_{\ell^{\prime}}=L$. In this case, it is clear that each $F_{i}$, with $i<0$, is ad-nilpotent on $L$.

If $A$ is any finite-dimensional Lie algebra, then the Ado-Iwasawa Theorem (see [J. Chapter VI]) implies that $A$ embeds in some $L=\mathfrak{g l}_{n}$ and therefore we obtain a filtration of $L$ with $F_{-1}=0, F_{0}=A$ and $F_{1}=L$. Thus, it is clearly hopeless to attempt to classify all the bounded filtrations of the various $\mathfrak{g l}_{n}$, even if only up to isomorphism. Nevertheless, there is at least something that can be done.

Again, let $\mathcal{F}$ be a filtration of an arbitrary Lie algebra $L$. If $\mathcal{G}=\left\{G_{i} \mid i \in \mathbb{Z}\right\}$ is a second such filtration, we say that $\mathcal{G}$ contains $\mathcal{F}$, or $\mathcal{G}$ is larger than $\mathcal{F}$, if $G_{i} \supseteq F_{i}$ for all $i$. In particular, it makes sense to speak about maximal bounded filtrations, and it is the goal of this paper to classify such filtrations when $L$ is a complex semisimple Lie algebra.

Maximal bounded filtrations were first introduced in the context of classifying the maximal graded subalgebras of affine Kac-Moody algebras, and the maximal

Received by the editors February 4, 2004.

2000 Mathematics Subject Classification. Primary 17B20, 17B70, 16W70.

The first author's research was carried out while visiting the University of Wisconsin-Madison, Imperial College and the University of Kent. He thanks all three mathematics departments.

The second author's research was supported in part by NSA grant 144-LQ65.

(C)2005 American Mathematical Society Reverts to public domain 28 years from publication 
graded subalgebras of loop toroidal Lie algebras. Recall that an affine Kac-Moody algebra can be realized as a central extension, by a 1-dimensional center, of the Lie algebra $L \otimes_{\mathbb{C}} \mathbb{C}[t, 1 / t]$, where $L$ is a finite-dimensional complex simple Lie algebra and $\mathbb{C}[t, 1 / t]$ is the complex group algebra of the infinite cyclic group $\langle t\rangle$. Note that $L \otimes_{\mathbb{C}} \mathbb{C}[t, 1 / t]$ is naturally a $\mathbb{Z}$-graded Lie algebra. The following theorem was obtained by Shalev, Zelmanov and the first author. It is stated in a sharpened form, based on an observation from $[\mathrm{B}$.

Theorem 1.1 ([BSZ $)$. Let $L$ be a finite-dimensional central simple Lie algebra over an arbitrary field $K$, and let $M=\bigoplus \sum_{i \in \mathbb{Z}} M_{i} t^{i}$ be a maximal $\mathbb{Z}$-graded subalgebra of $L \otimes_{K} K[t, 1 / t]$. Then one of the following holds:

(i) There exist a prime $p$ and $a \mathbb{Z} / p \mathbb{Z}$-grading $L=L_{0} \oplus L_{1} \oplus \cdots \oplus L_{p-1}$ of $L$, such that $M_{i}=L_{i \bmod p}$, for all $i \in \mathbb{Z}$.

(ii) There exists a maximal subalgebra $H$ of $L$ such that $M=H \otimes_{K} K[t, 1 / t]$.

(iii) The set $\left\{M_{i} \mid i \in \mathbb{Z}\right\}$ is a maximal bounded filtration of $L$.

We remark that $L$ is central simple if it is a simple Lie algebra over $K$ and if

$$
\left\{T \in \mathrm{GL}_{K}(L) \mid T[x, y]=[T x, y]=[x, T y] \text { for all } x, y \in L\right\},
$$

its centroid, is equal to $K$. It is known, by [J, Theorem X.1.3], that such Lie algebras remain central simple under all field extensions. Furthermore, if $L$ is merely assumed to be simple, then [J, Theorem X.1.2] asserts that its centroid is necessarily a field containing $K$. In particular, if $L$ is a finite-dimensional simple Lie algebra and if $K$ is algebraically closed, then $L$ is central simple.

Now, if $K=\mathbb{C}$ is the field of complex numbers, then the cyclic gradings of such Lie algebras $L$ correspond to automorphisms of finite order, and these were classified by Kac; see $[\mathrm{K}$. Furthermore, the maximal subalgebras of such Lie algebras were characterized in Dynkin's papers [D1, D2. Therefore, the classification of maximal graded subalgebras of affine Kac-Moody algebras will be completed once all maximal bounded filtrations of simple complex Lie algebras are known, and the results of the present paper achieve this in almost all cases.

Finally, B] studied the maximal graded subalgebras of loop toroidal Lie algebras and reduced their classification to the determination of certain maximal bounded filtrations indexed by finitely generated additive subgroups of $\mathbb{R}$. Because of this, we also briefly consider filtrations with respect to general Archimedean ordered groups. These groups are, of course, all additive subgroups of the real numbers $\mathbb{R}$ and hence are either dense or isomorphic to $\mathbb{Z}$. The following lemma applies equally well to filtrations over dense subgroups, and with the same proof, but we state it only for filtrations indexed by the group $\mathbb{Z}$.

Lemma 1.2. Let $L$ be a finite-dimensional semisimple Lie algebra and let $\mathcal{F}=$ $\left\{F_{i} \mid i \in \mathbb{Z}\right\}$ be a bounded filtration of $L$. Then $\mathcal{F}$ is contained in a maximal bounded filtration.

Proof. Let $\operatorname{dim}_{K} L=d$ and let $\mathcal{F}=\left\{F_{i} \mid i \in \mathbb{Z}\right\}$ be given with $F_{a}=L$ for some $a \geq 1$. If $\mathcal{G}=\left\{G_{i} \mid i \in \mathbb{Z}\right\}$ is any bounded filtration containing $\mathcal{F}$, then we also have $G_{a}=L$. Consider the chain of subspaces of $L$ given by

$$
G_{0} \supseteq G_{-a} \supseteq G_{-2 a} \supseteq \cdots \supseteq G_{-d a} .
$$

Since there are $d+1$ members of this chain and since $\operatorname{dim}_{K} L=d$, it follows that either they are all distinct and hence $G_{-d a}=0$, or two adjacent members are equal. 
In the latter case, say $G_{-(i-1) a}=G_{-i a}$ for some $i \geq 1$. Since $G_{a}=L$, it follows that $\left[L, G_{-i a}\right]=\left[G_{a}, G_{-i a}\right] \subseteq G_{-(i-1) a}=G_{-i a}$, and therefore $G_{-i a}$ is a Lie ideal of $L$. But the subscript $-i a$ is negative and $\mathcal{G}$ is bounded, so it is clear that $G_{-i a}$ is ad-nilpotent on $L$ and hence on itself. In other words, $G_{-i a}$ is a nilpotent ideal of $L$ and, since $L$ is semi-simple, we conclude that $G_{-i a}=0$. Thus $G_{-d a}=0$ in all cases and, with this, Zorn's Lemma clearly yields the result.

Of course, filtrations often arise from gradings, and bounded filtrations arise from finite gradings. Recall that $L=\bigoplus \sum_{i \in \mathbb{Z}} L_{i}$ is a $\mathbb{Z}$-grading of the Lie algebra if each $L_{i}$ is a $K$-subspace with $\left[L_{i}, L_{j}\right] \subseteq L_{i+j}$ for all $i, j$. Furthermore, this grading is said to be finite if only finitely many of the components $L_{i}$ are nonzero. In this case, it is clear that each $L_{i}$ with $i \neq 0$ is ad-nilpotent on $L$. Now any such grading determines an associated $\mathbb{Z}$-filtration $\mathcal{F}=\left\{F_{i} \mid i \in \mathbb{Z}\right\}$ by defining $F_{i}=\sum_{j \leq i} L_{j}$, and we have

Lemma 1.3. Let $L$ be a semisimple Lie algebra with a finite $\mathbb{Z}$-grading given by $L=\bigoplus \sum_{i} L_{i}$. If $\mathcal{F}=\left\{F_{i} \mid i \in \mathbb{Z}\right\}$ is the filtration associated to this grading, then $\mathcal{F}$ is a maximal bounded filtration.

Proof. By assumption, only finitely many of the components $L_{i}$ are nonzero. Hence, since $F_{i}=\sum_{j \leq i} L_{j}$, it is clear that $\mathcal{F}$ is at least a bounded filtration. Suppose, by way of contradiction, that $\mathcal{G}=\left\{G_{i} \mid i \in \mathbb{Z}\right\}$ is a bounded filtration properly larger than $\mathcal{F}$, and define $X_{i}=G_{i-1} \cap L_{i}$. Of course, only finitely many of these can be nonzero. Furthermore, since $L_{k} \subseteq F_{k} \subseteq G_{k}$, we see that $\left[L_{k}, X_{i}\right] \subseteq X_{i+k}$ and hence $X=\sum_{i} X_{i}$ is a Lie ideal of $L$.

We claim that $X$ is not zero. Indeed, since $\mathcal{G}$ is properly larger than the filtration $\mathcal{F}$, it follows that $G_{t}$ is properly larger than $F_{t}$ for some $t$, and hence we can choose $z \in L$ with $z \in G_{t} \backslash F_{t}$. Write $z=\sum_{i} z_{i} \in \sum_{i} L_{i}$ as the sum of its homogeneous components, and let $n$ be the maximal subscript with $z_{n} \neq 0$. Since $z \notin F_{t}$, we must have $n>t$. Then $z \in G_{n-1}$ and $z-z_{n} \in \sum_{i \leq n-1} L_{i}=F_{n-1} \subseteq G_{n-1}$, so $0 \neq z_{n} \in G_{n-1} \cap L_{n}=X_{n} \subseteq X$, as required.

Next, we claim that $X$ is Lie nilpotent. To this end, let $a$ be an integer with $L_{i}=0$ for all $i>a$, let $b$ be an integer with $G_{j}=0$ for all $j<b$, and let $\left[X_{i_{1}}, X_{i_{2}}, \ldots, X_{i_{n}}\right]_{\alpha}$ be any $n$-fold Lie product. Since $X_{i} \subseteq L_{i}$, it is clear that the $n$-fold product is contained in $L_{s}$ where $s=i_{1}+i_{2}+\cdots+i_{n}$. On the other hand, since $X_{i} \subseteq G_{i-1}$, we see that the product is also contained in $G_{s-n}$. In particular, if this product is nonzero, then we must have $s \leq a$ and $s-n \geq b$. Thus $n-s \leq-b$ and, by adding the two inequalities, we have $n \leq a-b$. It follows that $X$ is a nonzero nilpotent Lie ideal of $L$ of nilpotence index $\leq a-b+1$, and this contradicts the semisimplicity assumption. Thus, $\mathcal{F}$ is indeed maximal.

It is clear that the last paragraph of the above argument is simpler and more natural than the corresponding ring-theoretic proof in $[\mathrm{P}$, Lemma 1.2].

The previous two results are easily seen to be false without some sort of semisimplicity assumption. Indeed, suppose $L=L_{0} \oplus L_{1}$, where $L_{0}$ is a Lie subalgebra and $L_{1}$ is a nonzero abelian ideal. Then this is a finite grading of $L$, and its associated filtration $\mathcal{F}$ satisfies $F_{-1}=0, F_{0}=L_{0}$, and $F_{1}=L$. But $\mathcal{F}$ is not maximal since its 0 -term can certainly be extended to all of $L$. Furthermore, if $L_{0}$ is semisimple and if $\mathcal{G}$ is the filtration of $L$ given by $G_{-1}=0$ and $G_{0}=L$, then $\mathcal{G}$ is not contained in a maximal bounded filtration. Indeed, it is easy to see that any bounded filtration 
of $L$ containing $\mathcal{G}$ can be extended to a filtration having arbitrarily many negative terms equal to the ideal $L_{1}$.

Finally, suppose $L=A \oplus B$ is a direct sum of the two Lie algebras $A$ and $B$. If $\mathcal{A}=\left\{A_{i} \mid i \in \mathbb{Z}\right\}$ and $\mathcal{B}=\left\{B_{i} \mid i \in \mathbb{Z}\right\}$ are bounded filtrations of $A$ and $B$, respectively, and if we define $L_{i}=A_{i} \oplus B_{i} \subseteq L$, then $\mathcal{L}=\left\{L_{i} \mid i \in \mathbb{Z}\right\}$ is easily seen to be a bounded filtration of $L$. For convenience, we write $\mathcal{L}=\mathcal{A} \oplus \mathcal{B}$ and we say that $\mathcal{L}$ is the sum of $\mathcal{A}$ and $\mathcal{B}$. With this notation, we have

Lemma 1.4. Let $L$ be a Lie algebra and let $\mathcal{F}=\left\{F_{i} \mid i \in \mathbb{Z}\right\}$ be a bounded $\mathbb{Z}$-filtration of $L$.

(i) Suppose $L=A \oplus B$ is a direct sum of the $K$-Lie algebras $A$ and $B$. Then $\mathcal{L}$ is maximal if and only if $\mathcal{L}=\mathcal{A} \oplus \mathcal{B}$ with $\mathcal{A}$ and $\mathcal{B}$ maximal bounded filtrations of $A$ and $B$, respectively.

(ii) Suppose $L$ is a finite-dimensional complex semisimple Lie algebra, and write $L=L_{1} \oplus L_{2} \oplus \cdots \oplus L_{k}$ as a finite direct sum of simple Lie algebras. Then $\mathcal{F}$ is maximal if and only if it is a sum $\mathcal{F}=\mathcal{F}_{1} \oplus \mathcal{F}_{2} \oplus \cdots \oplus F_{k}$, where each $\mathcal{F}_{j}$ is a maximal bounded filtration of $L_{j}$.

Proof. (i) Let $\alpha: L \rightarrow A$ and $\beta: L \rightarrow B$ denote the natural projections, and let $\mathcal{F}=$ $\left\{F_{i} \mid i \in \mathbb{Z}\right\}$ be given. If $A_{i}=\alpha\left(F_{i}\right) \cong\left(F_{i}+B\right) / B$ and if $B_{i}=\beta\left(F_{i}\right) \cong\left(F_{i}+A\right) / A$, then it is easy to see that $\mathcal{A}=\left\{A_{i} \mid i \in \mathbb{Z}\right\}$ and $\mathcal{B}=\left\{B_{i} \mid i \in \mathbb{Z}\right\}$ are bounded filtrations of $A$ and $B$, respectively. Furthermore, it is clear that $F_{i} \subseteq A_{i} \oplus B_{i}$, so $\mathcal{F} \subseteq \mathcal{A} \oplus \mathcal{B}$. In particular, if $\mathcal{F}$ is maximal, then $\mathcal{F}=\mathcal{A} \oplus \mathcal{B}$, and clearly both $\mathcal{A}$ and $\mathcal{B}$ are maximal.

Conversely, suppose $\mathcal{F}^{\prime}=\mathcal{A}^{\prime} \oplus \mathcal{B}^{\prime}$ is a sum of maximal bounded filtrations, and let $\mathcal{F} \supseteq \mathcal{F}^{\prime}$ be arbitrary. Since $\mathcal{F} \subseteq \mathcal{A} \oplus \mathcal{B}$, by the result of the previous paragraph, we have $\mathcal{A} \supseteq \mathcal{A}^{\prime}$ and $\mathcal{B} \supseteq \mathcal{B}^{\prime}$. But $\mathcal{A}^{\prime}$ and $\mathcal{B}^{\prime}$ are maximal, so we must have equality throughout. In particular, $\mathcal{F}=\mathcal{F}^{\prime}$, and $\mathcal{F}^{\prime}$ is maximal.

(ii) This follows from the structure of finite-dimensional semisimple Lie algebras (see [ $\mathrm{Hu}$, Theorem 5.2]) and the obvious extension of part (i) above to finite direct sums of Lie algebras.

Thus, the study of maximal bounded filtrations in semisimple complex Lie algebras immediately reduces to the simple case. Because of this fact, we are mostly concerned in this paper with the simple Lie algebras, namely the algebras of type $A_{n}, B_{n}, C_{n}, D_{n}, E_{6}, E_{7}, E_{8}, F_{4}$ and $G_{2}$.

Our main result is essentially given below. More precise versions of each part can be found in later sections of this paper.

Theorem 1.5. Let $L$ be a finite-dimensional complex simple Lie algebra and let $\mathcal{F}=\left\{F_{i} \mid i \in \mathbb{Z}\right\}$ be a bounded filtration. Suppose $H$ is a Cartan subalgebra of $L$, write $V$ for the root space of $L$, and let $\widehat{V}=\operatorname{Hom}(V, \mathbb{R})$ denote its dual space.

(i) If $\mathcal{F}$ is maximal and if $L$ is of type $A_{n}, B_{n}, C_{n}$ or $D_{n}$, then $F_{0}$ contains a Cartan subalgebra of $L$.

(ii) The maximal filtrations $\mathcal{F}$ with $F_{0} \supseteq H$ are in one-to-one correspondence with the linear functionals $\lambda$ in a certain subset $\mathfrak{M}$ of $\widehat{V}$.

(iii) $\mathfrak{M}$ is the set of all functionals that take on integer values on some basis of $V$ consisting of roots of $L$. In particular, $\mathfrak{M}$ is a finite union of subgroups of $\widehat{V}$, each isomorphic to $\mathbb{Z}^{n}$, where $n=\operatorname{dim}_{\mathbb{R}} V$. 
We suspect that part (i) above is also true for the exceptional Lie algebras $E_{6}$, $E_{7}, E_{8}, F_{4}$ and $G_{2}$. While we have not been able to prove this, we do include a section of general lemmas which are, at the very least, suggestive.

For (ii), we use the root space decomposition of $L$ with respect to the Cartan subalgebra $H$. If $\lambda$ is any linear functional on $V$, then $\lambda$ defines a filtration $\mathcal{F}_{\lambda}$ of $L$ with $H$ contained in its 0 -term. Furthermore, any maximal filtration with $H$ in the 0-term must be of this type. It is not true, in general, that $\mathcal{F}_{\lambda}=\mathcal{F}_{\mu}$ if and only if $\lambda=\mu$. However, when the filtration is maximal, then $\mathcal{F}_{\lambda}$ uniquely determines $\lambda$, and hence we obtain the one-to-one correspondence.

Finally, in (iii), if $L$ is of type $A_{n}$, then $\mathfrak{M}$ is the set of all linear functionals that take on integer values on the roots $\Phi$ of $L$. If $L$ is of type $B_{n}, C_{n}$ or $D_{n}$, then $\mathfrak{M}$ is essentially (but not quite) the set of all functionals that take on integer or half integer values on the roots. The remaining types can be dealt with via computer computations. We include a tabulation at least for $G_{2}$ and $F_{4}$. There are also general results that can be read from the description of an arbitrary root in terms of a root basis. In particular, the description of the highest root, as given in $\mathrm{BO}$, Plates I-IX], yields some interesting information about $\mathfrak{M}$.

\section{MATRIX RINGS AND MAXIMAL FILTRATIONS}

Let $R$ be an associative $K$-algebra with 1 . A $\mathbb{Z}$-filtration $\mathcal{G}=\left\{G_{i} \mid i \in \mathbb{Z}\right\}$ of $R$ is a collection of $K$-subspaces

$$
\cdots \subseteq G_{-2} \subseteq G_{-1} \subseteq G_{0} \subseteq G_{1} \subseteq G_{2} \subseteq \cdots
$$

indexed by the integers $\mathbb{Z}$ such that $G_{i} G_{j} \subseteq G_{i+j}$ for all $i, j \in \mathbb{Z}$. One usually also assumes that $\bigcup_{i} G_{i}=R$ and $\bigcap_{i} G_{i}=0$. As with Lie algebras, we say that the filtration is bounded if there exist integers $\ell$ and $\ell^{\prime}$ with $G_{\ell}=0$ and $G_{\ell^{\prime}}=R$. Furthermore, if $\mathcal{G}^{\prime}=\left\{G_{i}^{\prime} \mid i \in \mathbb{Z}\right\}$ is a second such filtration, we say that $\mathcal{G}^{\prime}$ contains $\mathcal{G}$, or $\mathcal{G}^{\prime}$ is larger than $\mathcal{G}$, if $G_{i}^{\prime} \supseteq G_{i}$ for all $i$. In particular, it makes sense to speak about maximal bounded filtrations. We remark that the definition of filtration here differs slightly from that of $[\mathrm{P}$ where arbitrary rings were considered. In that case, the $G_{i}$ are merely assumed to be additive subgroups of $R$. However, in view of $[\mathrm{P}$, Lemma 2.1], there is no difference when dealing with maximal bounded filtrations.

Now suppose that $R=\mathrm{M}_{m}(K)$ and let $L$ be a Lie subalgebra of $\mathfrak{g l}_{m}(K)$. The goal of this section is to compare the filtrations of $R$ to those of $L$. We then apply known results for $R$ to obtain corresponding information about $L$. In particular, we will show that if $L$ is of type $A_{n}, B_{n}, C_{n}$ or $D_{n}$, and if $\mathcal{F}=\left\{F_{i} \mid i \in \mathbb{Z}\right\}$ is a maximal bounded filtration of $L$, then $F_{0}$ contains a Cartan subalgebra of $L$. To start with, we have

Lemma 2.1. Let $\mathcal{G}=\left\{G_{i} \mid i \in \mathbb{Z}\right\}$ be a bounded filtration of $R=\mathrm{M}_{m}(K)$ and let $L \subseteq \mathfrak{g l}_{m}(K)$. If $\mathcal{G}_{L}=\left\{F_{i} \mid i \in \mathbb{Z}\right\}$ is defined by $F_{i}=G_{i} \cap L$, then $\mathcal{G}_{L}$ is a bounded filtration of $L$.

Proof. If $x, y \in L \subseteq R$, then $[x, y]=x y-y x$ since $L$ is a Lie subalgebra of $\mathfrak{g l}_{m}(K)$. In particular, $\left[F_{i}, F_{j}\right] \subseteq F_{i} F_{j}+F_{j} F_{i} \subseteq G_{i} G_{j}+G_{j} G_{i} \subseteq G_{i+j}$, and thus $\left[F_{i}, F_{j}\right] \subseteq G_{i+j} \cap L=F_{i+j}$, as required.

Conversely, suppose $\mathcal{F}=\left\{F_{i} \mid i \in \mathbb{Z}\right\}$ is a filtration of $L$. Then we extend $\mathcal{F}$ to the ring $R$ by defining $\mathcal{F}^{R}=\left\{\widetilde{F}_{a} \mid a \in \mathbb{Z}\right\}$ to be the family of subspaces given by

$$
\widetilde{F}_{a}=\sum F_{i_{1}} F_{i_{2}} \cdots F_{i_{s}},
$$


where the sum is over all $s \geq 0$ and all subscripts with $i_{1}+i_{2}+\cdots+i_{s} \leq a$. Of course, when $s=0$ the subscript sum is 0 and the empty product is equal to $K$. The following is a key observation.

Lemma 2.2. Let $L$ be a Lie subalgebra of $\mathfrak{g l}_{m}(K)$ and assume that $L$ generates $\mathrm{M}_{m}(K)$ as a $K$-algebra. Assume further that every $x \in L$ which is ad-nilpotent on $\mathfrak{g l}_{m}(K)$ is necessarily a nilpotent matrix. If $\mathcal{F}=\left\{F_{i} \mid i \in \mathbb{Z}\right\}$ is a bounded filtration of $L$, then $\mathcal{F}^{R}=\left\{\widetilde{F}_{a} \mid a \in \mathbb{Z}\right\}$ is a bounded filtration of $\mathrm{M}_{m}(K)$.

Proof. It is clear that $\widetilde{F}_{a} \widetilde{F}_{b} \subseteq \widetilde{F}_{a+b}$ for all $a, b \in \mathbb{Z}$. Also, by assumption, $L$ generates $\mathrm{M}_{m}(K)$ and, since $\operatorname{dim}_{K} \mathrm{M}_{m}(K)<\infty$, we have $\mathrm{M}_{m}(K)=L^{0}+L^{1}+\cdots+L^{t}$ for some $t \geq 1$. In particular, if $F_{\ell^{\prime}}=L$ with $\ell^{\prime}>0$, then $\widetilde{F}_{\ell^{\prime} t}=\mathrm{M}_{m}(K)$. Next, let $x \in F_{-1}$ and note that $x$ is ad-nilpotent on $L$. Since $\mathrm{M}_{m}(K)=L^{0}+L^{1}+\cdots+L^{t}$, where $L^{0}=K$, and since ad $x$ is a derivation on $\mathrm{M}_{m}(K)$, it follows that $x$ is adnilpotent on $\mathfrak{g l}_{m}(K)$. Thus, by assumption, $x$ is a nilpotent matrix. We can now apply Engel's Theorem (see [Hu, Corollary 3.3]) to the Lie subalgebra $F_{-1}$ to deduce that there exists a flag $0=W_{0} \subset W_{1} \subset \cdots \subset W_{m}=K^{m}$ with $F_{-1} W_{i} \subseteq W_{i-1}$. In particular, $F_{-1}^{m}=0$.

Since $\mathcal{F}$ is bounded, we know that $F_{\ell}=0$ for some $\ell<0$. We show by induction on $s$ that if $F_{i_{1}} F_{i_{2}} \cdots F_{i_{s}} \neq 0$, then $m \ell<i_{1}+i_{2}+\cdots+i_{s}$. This is clear for $s=0$ where, by assumption, the subscript sum is 0 and the empty product is $K$, or for $s=1$, since $i_{1}>\ell$. Now let $s \geq 2$ and note that $F_{i} F_{j} \subseteq F_{j} F_{i}+\left[F_{i}, F_{j}\right] \subseteq$ $F_{j} F_{i}+F_{i+j}$ since $L \subseteq \mathfrak{g l}_{m}(K)$. In particular, if we interchange two adjacent factors in $F_{i_{1}} F_{i_{2}} \cdots F_{i_{s}} \neq 0$, say $F_{i_{r}}$ and $F_{i_{r+1}}$, then either

$$
F_{i_{1}} \cdots F_{i_{r-1}} F_{i_{r+1}} F_{i_{r}} \cdots F_{i_{s}} \neq 0
$$

or

$$
F_{i_{1}} \cdots F_{i_{r-1}} F_{i_{r}+i_{r+1}} \cdots F_{i_{s}} \neq 0
$$

or both. If the latter occurs, then $m \ell<i_{1}+i_{2}+\cdots+i_{s}$ by induction on $s$. Thus, we can assume throughout that the former occurs, and this enables us to put the subscripts in their natural order.

In other words, we now have $F_{\ell+1}^{k_{\ell+1}} \cdots F_{-1}^{k_{-1}} F_{0}^{k_{0}} \cdots F_{n}^{k_{n}} \neq 0$ and in particular $0 \neq F_{\ell+1}^{k_{\ell+1}} \cdots F_{-1}^{k_{-1}} \subseteq F_{-1}^{k}$, where $k=k_{-1}+k_{-2}+\cdots+k_{\ell+1}$. Since $F_{-1}^{m}=0$, it follows that $k<m$, so $k \ell>m \ell$ and hence

$$
i_{1}+i_{2}+\cdots+i_{s} \geq(-1) k_{-1}+(-2) k_{-2}+\cdots+(\ell+1) k_{\ell+1} \geq k \ell>m \ell .
$$

This proves the inductive result, and the definition of $\widetilde{F}_{a}$ now implies that $\widetilde{F}_{m \ell}=0$. In other words, $\mathcal{F}^{R}$ is a bounded filtration of $R$.

In order to apply the above, we need the following well-known observation.

Lemma 2.3. Let $x \in \mathrm{M}_{m}(K)$ and assume that $x$ is ad-nilpotent in its action on $\mathfrak{g l}_{m}(K)$. If $\bar{K}$ is the algebraic closure of $K$, then $x=k+z$ where $k \in \bar{K}$ is a scalar matrix and $z \in \mathrm{M}_{m}(\bar{K})$ is nilpotent. Furthermore:

(i) If $K$ is a perfect field, then $k \in K$ and $z \in \mathrm{M}_{m}(K)$.

(ii) If $\operatorname{tr} x=0$ and $m \neq 0$ in $K$, then $x=z$ is nilpotent.

(iii) If $*$ is an involution on $\mathrm{M}_{m}(K)$ fixing $K$ elementwise and if char $K \neq 2$, then $x^{*}=-x$ implies that $x=z$ is nilpotent. 
Proof. If $\bar{K}$ is the algebraic closure of $K$, then $\mathrm{M}_{m}(\bar{K}) \supseteq \mathrm{M}_{m}(K)$ and it is clear that $x$ is ad-nilpotent on $\mathfrak{g l}_{m}(\bar{K})$. Suppose $x$ has at least two distinct eigenvalues, say $\alpha$ and $\beta$, and let $v_{\alpha}$ and $v_{\beta}$ be corresponding eigenvectors. If $u \in \mathrm{M}_{m}(\bar{K})$ with $u v_{\alpha}=\lambda v_{\beta}$ for some $\lambda \in \bar{K}$, then

$$
(u \operatorname{ad} x) v_{\alpha}=(u x-x u) v_{\alpha}=u \alpha v_{\alpha}-x \lambda v_{\beta}=\lambda(\alpha-\beta) v_{\beta} .
$$

In particular, if $y \in \mathfrak{g l}_{m}(\bar{K})$ with $y v_{\alpha}=v_{\beta}$, then $\left(y(\operatorname{ad} x)^{i}\right) v_{\alpha}=(\alpha-\beta)^{i} v_{\beta}$, and hence $(\operatorname{ad} x)^{i} \neq 0$ for all $i$, contrary to our assumption. It follows that $x$ has only one eigenvalue $k \in \bar{K}$, and therefore $x=k+z$ with $z$ nilpotent.

(i) Since $k$ is the unique root of the characteristic polynomial of $x$, we conclude that $k \in K$ if $K$ is a perfect field.

(ii) If $\operatorname{tr} x=0$, then $m k=0$ and therefore $k=0$ when $m \neq 0$ in $K$.

(iii) Since $*$ fixes $K$ elementwise, it is clear that $*$ extends to an involution on $\mathrm{M}_{m}(\bar{K})=\bar{K} \otimes_{K} \mathrm{M}_{m}(K)$. Now suppose $x^{*}=-x$. Since $x-k$ is a singular matrix, so also is $(x-k)^{*}=-x-k=-(x+k)$. Hence $-k=k$ and again $k=0$.

As an example, we have

Lemma 2.4. Let $K$ be an algebraically closed field, let $R=\mathrm{M}_{m}(K)$, and let $L$ be a Lie subalgebra of $\mathfrak{g l}_{m}(K)$ acting irreducibly on $K^{m}$. Suppose $L=[L, L]$ and that $m \neq 0$ in $K$. If $\mathcal{F}=\left\{F_{i} \mid i \in \mathbb{Z}\right\}$ is a bounded $\mathbb{Z}$-filtration of $L$, then $\mathcal{F}^{R}$ is a bounded $\mathbb{Z}$-filtration of $R$.

Proof. Since $K^{m}$ is an irreducible $L$-module, the finite-dimensional associative $K$ algebra $\langle L\rangle$ generated by $L$ is a primitive subring of $R$. Thus, since $K$ is algebraically closed, it follows that $\langle L\rangle=R$. Furthermore, the assumption $L=[L, L]$ implies that $L \subseteq \mathfrak{s l}_{m}(K)$ and hence that $\operatorname{tr} L=0$. In view of part (ii) of the preceding lemma, we see that if $x \in L$ is ad-nilpotent on $\mathfrak{g l}_{m}(K)$, then $x$ is a nilpotent matrix. Lemma 2.2 now yields the result.

Our main applications of this correspondence between the filtrations of $L$ and those of $R$ are given below, where we use the results of the second author in $[\mathrm{P}$. We start with the special linear Lie algebra.

Proposition 2.5. Let $K$ be a field, let $L=\mathfrak{s l}_{m}(K)$ with $m \geq 2$, and suppose that $\mathcal{F}=\left\{F_{i} \mid i \in \mathbb{Z}\right\}$ is a maximal bounded filtration of $L$. If $m \neq 0$ in $K$, then $F_{0}$ contains a Cartan subalgebra of $L$.

Proof. If $R=\mathrm{M}_{m}(K)$ with $m \geq 2$, then it is clear that $L=\mathfrak{s l}_{m}(K)$ generates $R$ as an associative $K$-algebra. Furthermore, if $x \in L$ is ad-nilpotent on $\mathfrak{g l}_{m}(K)$, then it follows from Lemma 2.3(ii) that $x$ is a nilpotent matrix. We conclude from Lemma 2.2 that $\mathcal{F}^{R}=\left\{\widetilde{F}_{i} \mid i \in \mathbb{Z}\right\}$ is a bounded filtration of $R$, and [P, Lemma 1.1] implies that $\mathcal{F}^{R}$ extends to a maximal bounded filtration $\mathcal{G}=\left\{G_{i} \mid i \in \mathbb{Z}\right\}$ of $R$.

In view of Lemma 2.1. $\mathcal{G}_{L}=\left\{G_{i} \cap L \mid i \in \mathbb{Z}\right\}$ is a bounded filtration of $L$, and note that $G_{i} \cap L \supseteq \widetilde{F}_{i} \cap L \supseteq F_{i}$. Thus, $\mathcal{G}_{L} \supseteq \mathcal{F}$ and, since $\mathcal{F}$ is a maximal bounded filtration of $L$, we conclude that $\mathcal{G}_{L}=\mathcal{F}$. In particular, $G_{0} \cap L=F_{0}$. But $G_{0}$ contains a full diagonal $D=\mathrm{D}_{m}(K)$, by [P, Lemma 2.5], and hence we see that $F_{0}=G_{0} \cap L$ contains $H=D \cap L=\{y \in D \mid \operatorname{tr} y=0\}$.

Finally, note that $\mathfrak{g l}_{m}(K)$ is a direct sum of 1-dimensional ad $D$-modules, and hence $L$ is a direct sum of 1 -dimensional ad $H$-modules. Furthermore, it is easy to see that $\mathfrak{C}_{L}(H)=H$ and therefore the complete reducibility of the action of 
ad $H$ on $L$ implies that $\mathfrak{N}_{L}(H)=\mathfrak{C}_{L}(H)=H$. Thus $H \subseteq F_{0}$ is indeed a Cartan subalgebra of $L$.

Now let $*$ be an involution of $\mathrm{M}_{m}(K)$ of the first kind, so that $*$ acts trivially on $K$. As is well known, the set of all skew-symmetric matrices under the action of * is a Lie subalgebra of $\mathfrak{g l}_{m}(K)$. For such Lie algebras, we have

Proposition 2.6. Let $K$ be an algebraically closed field of characteristic $\neq 2$, let * be an involution of $\mathrm{M}_{m}(K)$ of the first kind, and let $L$ be the Lie subalgebra of $\mathfrak{g l}_{m}(K)$ consisting of all skew-symmetric matrices under the action of $*$. If $\mathcal{F}=\left\{F_{i} \mid i \in \mathbb{Z}\right\}$ is a maximal bounded filtration of $L$ and if $m \geq 3$, then $F_{0}$ contains a Cartan subalgebra of $L$.

Proof. Let $R=\mathrm{M}_{m}(K)$ and let $L$ be the Lie subalgebra of $\mathfrak{g l}_{m}(K)$ consisting of all skew-symmetric matrices under the action of $*$. Since $m \geq 3$, [He, Theorem 2.1.10] implies that $L$ generates $R$. Furthermore, if $x \in L$ is ad-nilpotent on $\mathfrak{g l}_{m}(K)$, then it follows from Lemma 2.3(iii) that $x$ is a nilpotent matrix. We therefore conclude from Lemma 2.2 that $\mathcal{F}^{R}=\left\{\widetilde{F}_{i} \mid i \in \mathbb{Z}\right\}$ is a bounded filtration of $R$, and we note that each $F_{i}$ is $*$-stable. Thus each $\widetilde{F}_{i}$ is also $*$-stable and, by $\left[\mathrm{P}\right.$, Lemma 1.1], $\mathcal{F}^{R}$ extends to a bounded $*$-stable filtration $\mathcal{G}=\left\{G_{i} \mid i \in \mathbb{Z}\right\}$ maximal in the collection of all $*$-stable bounded filtrations.

In view of Lemma $2.1 \mathcal{G}_{L}=\left\{G_{i} \cap L \mid i \in \mathbb{Z}\right\}$ is a bounded filtration of $L$, and note that $G_{i} \cap L \supseteq \widetilde{F}_{i} \cap L \supseteq F_{i}$. Thus, $\mathcal{G}_{L} \supseteq \mathcal{F}$ and, since $\mathcal{F}$ is a maximal bounded filtration of $L$, we conclude that $\mathcal{G}_{L}=\mathcal{F}$. In particular, $G_{0} \cap L=F_{0}$. By $[\mathrm{P}$, Lemma 5.7], we can write $1=e_{1}+e_{2}+\cdots+e_{m}$ as an orthogonal sum of primitive idempotents with $D=\mathrm{D}_{m}(K)=\sum_{i=1}^{m} K e_{i} \subseteq G_{0}$. Furthermore, $\left\{e_{1}, e_{2}, \ldots, e_{m}\right\}$ is a $*$-stable set containing at most one $*$-stable idempotent. In particular, $F_{0}=G_{0} \cap L$ contains $H=D \cap L=\sum_{i=1}^{m} K\left(e_{i}-e_{i}^{*}\right)$.

Finally, note that $\mathfrak{g l}_{m}(K)$ is a direct sum of 1-dimensional ad $D$-modules, and hence $L$ is a direct sum of 1 -dimensional ad $H$-modules. Furthermore, it is easy to see that $H$ is self centralizing in $L$. Indeed, if $e_{i} \neq e_{i}^{*}$, then the centralizer of $e_{i}-e_{i}^{*}$ in $R$ also commutes with $\left(e_{i}-e_{i}^{*}\right)^{2}=e_{i}+e_{i}^{*}$, and hence it centralizes both $e_{i}$ and $e_{i}^{*}$. Since $\left\{e_{1}, e_{2}, \ldots, e_{m}\right\}$ contains at most one idempotent fixed by $*$, it therefore follows easily that $\mathfrak{C}_{R}(H)=\mathfrak{C}_{R}(D)=D$ and hence that $\mathfrak{C}_{L}(H)=D \cap L=H$. The complete reducibility of the action of ad $H$ on $L$ now implies that $\mathfrak{N}_{L}(H)=$ $\mathfrak{C}_{L}(H)=H$, and $H \subseteq F_{0}$ is indeed a Cartan subalgebra of $L$.

The preceding two propositions now combine to yield

Corollary 2.7. Let $K$ be the field of complex numbers and let $L$ be a simple $K$-Lie algebra of type $A_{n}, B_{n}, C_{n}$ or $D_{n}$. If $\mathcal{F}=\left\{F_{i} \mid i \in \mathbb{Z}\right\}$ is a maximal bounded filtration of $L$, then $F_{0}$ contains a Cartan subalgebra of $L$.

Proof. The simple Lie algebras $L$ of type $A_{n}, B_{n}, C_{n}$ or $D_{n}$ are described in $\mathrm{Hu}$, Section 1.1]. Type $A_{n}$ is, of course, $\mathfrak{s l}_{n+1}(K)$, while $B_{n}, C_{n}$ and $D_{n}$ are defined to be the set of all matrices $x$ in $M_{m}(K)$ satisfying $s x=-x^{T} s$ where $x^{T}$ is the transpose of $x$. Here $m=2 n$ or $2 n+1$, and $s$ is a suitable fixed matrix depending upon the type. In particular, if $*: \mathrm{M}_{m}(K) \rightarrow \mathrm{M}_{m}(K)$ is given by $y^{*}=s^{-1} y^{T} s$, then $*$ is easily seen to be an involution of the matrix ring since $s^{T}= \pm s$, and $L$ is the set of all skew-symmetric matrices under the action of $*$. Propositions 2.5 and 2.6 now yield the result. 
The five remaining simple Lie algebras $L$, namely those of type $E_{6}, E_{7}, E_{8}, F_{4}$ and $G_{2}$, are not easily identified as subspaces of a suitable $\mathrm{M}_{m}(K)$. Thus, they have to be treated differently. In Section 7, we will discuss some techniques which may lead to a proof of the analog of Corollary 2.7 for these algebras.

\section{Cartan filtrations}

Let $L$ be a finite-dimensional simple Lie algebra over the complex numbers $K$. In this section, we consider filtrations $\mathcal{F}=\left\{F_{i} \mid i \in \mathbb{Z}\right\}$ where, of course, each $F_{i}$ is a $K$-subspace of $L$. For convenience, we say that a bounded $\mathbb{Z}$-filtration $\mathcal{F}$ of $L$ is Cartan if $F_{0}$ contains a Cartan subalgebra $L_{0}$ of $L$. Our goal here is to determine the maximal bounded $\mathbb{Z}$-filtrations of $L$ that are also Cartan. Note that, if $\mathcal{G}=\left\{G_{i} \mid i \in \mathbb{Z}\right\}$ is a filtration containing the filtration $\mathcal{F}$, then $G_{0} \supseteq F_{0} \supseteq L_{0}$ and hence $\mathcal{G}$ is also Cartan. Let $L=L_{0}+\sum_{\alpha \in \Phi} L_{\alpha}$ be the root space decomposition of $L$. Here $\Phi=\Phi(L)$ is the set of roots of $L$ and each $L_{\alpha}$ with $\alpha \in \Phi$ a 1-dimensional subspace of $L$. The basic tool here is the degree function $f$ of $\mathcal{F}$ defined by

$$
f(\alpha)=\min \left\{i \in \mathbb{Z} \mid L_{\alpha} \subseteq F_{i}\right\} .
$$

Since $\mathcal{F}$ is bounded, $f(\alpha)$ is a well-defined integer for all $\alpha \in \Phi$. For convenience, set $\Phi^{\prime}=\Phi \cup 0$ and define $f(0)=0$.

Lemma 3.1. Let $\mathcal{F}=\left\{F_{i} \mid i \in \mathbb{Z}\right\}$ be a Cartan filtration of the simple Lie algebra $L$ and let $f$ be its degree function.

(i) For each $i \in \mathbb{Z}$, we have $F_{i}=\sum L_{\alpha}$, where the sum is over all $\alpha \in \Phi^{\prime}$ with $f(\alpha) \leq i$. In particular, $f$ uniquely determines the filtration.

(ii) If $\alpha, \beta$ and $\alpha+\beta$ are contained in $\Phi$, then $f(\alpha)+f(\beta) \geq f(\alpha+\beta)$. Furthermore, $f(\alpha)+f(-\alpha) \geq 0$.

(iii) If $\mathcal{G}=\left\{G_{i} \mid i \in \mathbb{Z}\right\}$ is a second Cartan filtration of $L$ with degree function $g$, then $\mathcal{G}$ contains $\mathcal{F}$ if and only if $g(\alpha) \leq f(\alpha)$ for all $\alpha \in \Phi$.

Proof. (i) Since $F_{0} \supseteq L_{0}$, it follows that $\left[L_{0}, F_{i}\right] \subseteq F_{i}$ for all $i \in \mathbb{Z}$. Thus $F_{i}$ is an $L_{0}$-module under the adjoint action and hence each such $F_{i}$ is a direct sum of various $L_{\alpha}$ 's with $\alpha \in \Phi$ along with $F_{i} \cap L_{0}$. Note that $L_{\alpha} \subseteq F_{i}$ if and only if $i \geq f(\alpha)$. Furthermore, by assumption, $L_{0} \subseteq F_{0} \subseteq F_{i}$ for all $i \geq 0$. Also, because no nonzero element of $L_{0}$ can be ad-nilpotent on $L$, we must have $L_{0} \cap F_{i}=0$ if $i<0$. Since $f(0)=0$, the result is now clear.

(ii) Say $f(\alpha)=a$ and $f(\beta)=b$. Then $L_{\alpha} \subseteq F_{a}$ and $L_{\beta} \subseteq F_{b}$, so $\left[L_{\alpha}, L_{\beta}\right] \subseteq$ $\left[F_{a}, F_{b}\right] \subseteq F_{a+b}$. Since $\alpha+\beta \in \Phi$, we know that $\left[L_{\alpha}, L_{\beta}\right]=L_{\alpha+\beta}$ by $[\mathrm{Hu}$, Proposition 8.4]. Thus $L_{\alpha+\beta} \subseteq F_{a+b}$ and, by definition, we have $f(\alpha+\beta) \leq$ $a+b=f(\alpha)+f(\beta)$. Furthermore, $0 \neq\left[L_{\alpha}, L_{-\alpha}\right] \subseteq L_{0} \subseteq F_{0}$, so we also have $f(\alpha)+f(-\alpha) \geq f(0)=0$.

(iii) In view of the above, the inclusions $F_{i} \subseteq G_{i}$ for all $i \in \mathbb{Z}$ occur if and only if $L_{\alpha} \subseteq F_{i}$ implies that $L_{\alpha} \subseteq G_{i}$. Obviously, the latter occurs precisely when $f(\alpha) \geq g(\alpha)$ for all $\alpha \in \Phi$.

Now let $V$ be the real inner product space determined by $\Phi(L)$, the root set of $L$. If $\lambda: V \rightarrow \mathbb{R}$ is a linear functional, we define the filtration $\mathcal{F}_{\lambda}=\left\{F_{i} \mid i \in \mathbb{Z}\right\}$ by $F_{i}=\sum_{\alpha} L_{\alpha}$, where the sum is over all $\alpha \in \Phi^{\prime}$ with $\lambda(\alpha) \leq i$. Recall that if $a \in \mathbb{R}$ is a real number, then $\lceil a\rceil$ is the smallest integer bigger than or equal to $a$.

Lemma 3.2. If $\lambda: V \rightarrow \mathbb{R}$, then $\mathcal{F}_{\lambda}$ is a Cartan filtration of $L$ with degree function given by $f_{\lambda}(\alpha)=\lceil\lambda(\alpha)\rceil$. 
Proof. Fix $a, b \in \mathbb{Z}$, let $L_{\alpha}$ be a summand of $F_{a}$ and let $L_{\beta}$ be a summand of $F_{b}$. Then $\alpha, \beta \in \Phi^{\prime}$ and, by definition, we have $a \geq \lambda(\alpha)$ and $b \geq \lambda(\beta)$. If $\left[L_{\alpha}, L_{\beta}\right]=0$, then certainly $\left[L_{\alpha}, L_{\beta}\right] \subseteq F_{a+b}$. On the other hand, if $\left[L_{\alpha}, L_{\beta}\right] \neq 0$, then we know that $\gamma=\alpha+\beta \in \Phi^{\prime}$ and that $\left[L_{\alpha}, L_{\beta}\right] \subseteq L_{\gamma}$. But then $\lambda(\gamma)=\lambda(\alpha)+\lambda(\beta) \leq a+b$, so $L_{\gamma} \subseteq F_{a+b}$, and again we conclude that $\left[L_{\alpha}, L_{\beta}\right] \subseteq F_{a+b}$. Thus, $\mathcal{F}_{\lambda}$ is a filtration, and it is Cartan since $\lambda(0)=0$. Finally, $L_{\alpha} \subseteq F_{i}$ if and only if $i \geq \lambda(\alpha)$, so the minimum value of the integer $i$ is precisely $\lceil\lambda(\alpha)\rceil$.

Since $\mathcal{F}_{\lambda}$ is a $\mathbb{Z}$-filtration, it is clear that $\mathcal{F}_{\lambda}$ does not uniquely determine $\lambda$ in general, and certainly not all of these are maximal. Indeed, when we determine which of these dual filtrations are maximal, we will have to consider those $\lambda$ in the dual space $\widehat{V}$ of $V$ with $\lambda(\Phi) \subseteq \mathbb{Z}$ or perhaps with some other restriction on the values taken on. These specific questions will be discussed and answered in the next several sections. Now, we note

Lemma 3.3. $\mathcal{F}_{\lambda}$ is the filtration associated with a $\mathbb{Z}$-grading of $L$, having $L_{0}$ in the 0 -component, if and only if $\lambda(\Phi) \subseteq \mathbb{Z}$.

Proof. If $\lambda(\Phi) \subseteq \mathbb{Z}$, then $\mathcal{F}_{\lambda}$ comes from the $\mathbb{Z}$-grading obtained by giving $L_{\alpha}$ the grade $\lambda(\alpha)$. On the other hand, if $\mathcal{F}_{\lambda}$ comes from such a grading, then, since $L_{0}$ is in the 0-component, it follows as above that each component is a direct sum of suitable $L_{\alpha}$ with $\alpha \in \Phi^{\prime}$. But, for $\alpha \neq 0$, we have $0 \neq\left[L_{\alpha}, L_{-\alpha}\right] \subseteq L_{0}$, so the grade of $L_{\alpha}$ and of $L_{-\alpha}$ must be negatives of each other. This, in turn, implies that $\lceil\lambda(\alpha)\rceil=-\lceil\lambda(-\alpha)\rceil=-\lceil-\lambda(\alpha)\rceil=\lfloor\lambda(\alpha)\rfloor$, where the latter expression is the greatest integer in $\lambda(\alpha)$. Hence, $\lambda(\alpha) \in \mathbb{Z}$.

Now, if all roots in $\Phi$ have the same length, then we consider them to be short. Otherwise, there are two different lengths, the short roots and the long ones. For convenience, we let $\|\sigma\|$ denote the common length of a short root. If $\alpha \in \Phi$, we define $k(\alpha)$ to equal the number of short $\operatorname{roots} \beta$ with $(\alpha, \beta)<0$ and $\beta \neq \pm \alpha$. Next, we define

$$
d(\alpha)= \begin{cases}(k(\alpha)+4) \cdot(\|\alpha\| /\|\sigma\|)^{2}, & \text { if } \alpha \text { is a short root, } \\ k(\alpha) \cdot(\|\alpha\| /\|\sigma\|)^{2}, & \text { if } \alpha \text { is a long root. }\end{cases}
$$

Since the Weyl group acts transitively on the roots in $\Phi$ having the same length $[\mathrm{Hu}$, Lemma $10.4 \mathrm{C}]$, it is clear that the parameters $k(\alpha)$ and $d(\alpha)$ depend only upon the length of the root $\alpha \in \Phi(L)$. With this, and using the description of $V$ as given in [Bo, Plates I-IX], it is easy to determine the entries in the following table.

\begin{tabular}{|c|c|c|c|c|}
\hline & \multicolumn{2}{|c|}{ Short Root } & \multicolumn{2}{|c|}{ Long Root } \\
\hline Type & $k(\alpha)$ & $d(\alpha)$ & $k(\alpha)$ & $d(\alpha)$ \\
\hline$\overline{A_{n}}$ & $2(n-1)$ & $\overline{2(n+1)}$ & \multicolumn{2}{|c|}{$\overline{~ n o n e}$} \\
\hline$\overline{B_{n}}$ & $\overline{0}$ & 4 & $\overline{2}$ & 4 \\
\hline$C_{n}$ & $4(n-2)$ & $4(n-1)$ & $2(n-1)$ & $4(n-1)$ \\
\hline$D_{n}$ & $4(n-2)$ & $4(n-1)$ & \multicolumn{2}{|c|}{ none } \\
\hline$E_{6}$ & 20 & 24 & \multicolumn{2}{|c|}{ none } \\
\hline$E_{7}$ & 32 & 36 & \multicolumn{2}{|c|}{ none } \\
\hline$E_{8}$ & 56 & 60 & \multicolumn{2}{|c|}{ none } \\
\hline$F_{4}$ & 8 & 12 & 6 & 12 \\
\hline$G_{2}$ & 2 & 6 & 2 & 6 \\
\hline
\end{tabular}


As a consequence of the $B_{n}, C_{n}, F_{4}$ and $G_{2}$ computations above, we have

Lemma 3.4. If $L$ is a simple Lie algebra, then the parameter $d=d(\alpha)$ is the same for all roots $\alpha \in \Phi(L)$.

We will offer a partial explanation for this equality after Lemma 3.6 is proved. Next, we need

Lemma 3.5. Let $\alpha, \beta \in \Phi$ with $\alpha \neq \pm \beta$ and with $\beta$ a short root. If $(\alpha, \beta)<0$, then $\alpha+\beta$ is a short root in $\Phi,(\alpha, \beta)=-\|\alpha\|^{2} / 2$ and $(\alpha, \alpha+\beta)=-(\alpha, \beta)$.

Proof. Since $(\alpha, \beta)<0,[\mathrm{Hu}$, Lemma 9.4] implies that $\alpha+\beta$ is a root. Furthermore, using $\|\alpha\| \geq\|\beta\|$, we see that $2(\alpha, \beta) /\|\alpha\|^{2}=\langle\beta, \alpha\rangle=-1$. In particular, $(\alpha, \beta)=$ $-\|\alpha\|^{2} / 2$, and $\|\alpha+\beta\|^{2}=\|\alpha\|^{2}+2(\alpha, \beta)+\|\beta\|^{2}=\|\beta\|^{2}$. Thus $\alpha+\beta$ is short, and $(\alpha, \alpha+\beta)=\|\alpha\|^{2}+(\alpha, \beta)=-(\alpha, \beta)$, as required.

With this observation, we can now prove the following key lemma in a surprisingly easy manner. Here $d$ is the parameter given by Lemma 3.4 .

Lemma 3.6. Let $\mathcal{F}=\left\{F_{i} \mid i \in \mathbb{Z}\right\}$ be a Cartan filtration of the simple Lie algebra $L$. If $V$ is the root space of $L$, then there exists a linear functional $\lambda: V \rightarrow \mathbb{R}$ such that $\mathcal{F} \subseteq \mathcal{F}_{\lambda}$ and $\lambda(\Phi) \subseteq \mathbb{Z} / d$.

Proof. Let $f$ be the degree function of $\mathcal{F}$, let $\Psi$ denote the set of short roots in $\Phi$, and let $d>0$ be the common value of the $d(\alpha)$ as given in Lemma 3.4. Define the functional $\mu: V \rightarrow \mathbb{R}$ by

$$
\mu(v)=\sum_{\beta \in \Psi} 2(v, \beta) \cdot f(\beta) /\|\beta\|^{2}
$$

and let $\lambda: V \rightarrow \mathbb{R}$ be given by $\lambda=\mu / d$. We will show that $\mathcal{F} \subseteq \mathcal{F}_{\lambda}$.

To this end, fix $\alpha \in \Phi$ and let $\Psi(\alpha)$ denote the set of short roots $\beta$ with $\beta \neq \pm \alpha$ and with $(\alpha, \beta)<0$. Recall that the size of $\Psi(\alpha)$ is precisely equal to $k(\alpha)$. Furthermore, note that $\Psi^{*}(\alpha)=-\Psi(\alpha)$ is the set of short roots $\beta$ with $\beta \neq \pm \alpha$ and with $(\alpha, \beta)>0$. In particular, this set is disjoint from $\Psi(\alpha)$, and they both have the same size. By the preceding lemma, if $\beta \in \Psi(\alpha)$, then $\alpha+\beta \in \Psi^{*}(\alpha)$. Thus, by size considerations, the set $\alpha+\Psi(\alpha)$ of all such $\alpha+\beta$ is precisely equal to $\Psi^{*}(\alpha)$.

Now let $\beta \in \Psi(\alpha)$. Since $\alpha+\beta \in \Phi$ by Lemma 3.5, Lemma 3.1(ii) implies that $f(\alpha) \geq f(\alpha+\beta)-f(\beta)$. Thus, since $-(\alpha, \beta)=\|\alpha\|^{2} / 2>0$, we can multiply the previous inequality by this positive quantity to obtain

$$
\|\alpha\|^{2} \cdot f(\alpha) / 2 \geq(\alpha, \beta) \cdot f(\beta)-(\alpha, \beta) \cdot f(\alpha+\beta) .
$$

In particular, since $(\alpha, \alpha+\beta)=-(\alpha, \beta)$ by Lemma 3.5, this yields

$$
\|\alpha\|^{2} \cdot f(\alpha) / 2 \geq(\alpha, \beta) \cdot f(\beta)+(\alpha, \alpha+\beta) \cdot f(\alpha+\beta) .
$$

Hence, summing over all $\beta \in \Psi(\alpha)$, we get

$$
\|\alpha\|^{2} \cdot k(\alpha) \cdot f(\alpha) / 2 \geq \sum_{\beta \in \Psi(\alpha)}(\alpha, \beta) \cdot f(\beta)+\sum_{\beta \in \Psi(\alpha)}(\alpha, \alpha+\beta) \cdot f(\alpha+\beta) .
$$

As we indicated, the set $\alpha+\Psi(\alpha)$ is precisely equal to $\Psi^{*}(\alpha)$, so the second summation above is just $\sum_{\beta \in \Psi^{*}(\alpha)}(\alpha, \beta) \cdot f(\beta)$, and hence we have

$$
\|\alpha\|^{2} \cdot k(\alpha) \cdot f(\alpha) / 2 \geq \sum_{\beta \in \Psi(\alpha) \cup \Psi^{*}(\alpha)}(\alpha, \beta) \cdot f(\beta) .
$$


Now if $\alpha$ is a long root, then $\Psi(\alpha) \cup \Psi^{*}(\alpha)$ is the set of all short roots $\beta \in \Psi$ with $(\alpha, \beta) \neq 0$. Thus, the right-hand summand in $(*)$ is unchanged if we add the remaining short roots $\beta$, since all of these satisfy $(\alpha, \beta)=0$. Doing this, we then obtain

$$
\|\alpha\|^{2} \cdot k(\alpha) \cdot f(\alpha) / 2 \geq \sum_{\beta \in \Psi}(\alpha, \beta) \cdot f(\beta) .
$$

Thus, since $\|\beta\|^{2}=\|\sigma\|^{2}$ for all $\beta \in \Psi$, we have

$$
d(\alpha) \cdot f(\alpha)=k(\alpha) \cdot f(\alpha) \cdot(\|\alpha\| /\|\sigma\|)^{2} \geq \sum_{\beta \in \Psi} f(\beta) \cdot 2(\alpha, \beta) /\|\beta\|^{2}=\mu(\alpha)
$$

and hence $f(\alpha) \geq \mu(\alpha) / d(\alpha)=\mu(\alpha) / d=\lambda(\alpha)$.

On the other hand, if $\alpha$ is a short root, then there are two additional short roots $\beta$ with $(\alpha, \beta) \neq 0$, namely $\beta=\alpha$ and $\beta=-\alpha$. But certainly

$$
\|\alpha\|^{2} \cdot 2 \cdot f(\alpha) / 2 \geq(\alpha, \alpha) \cdot f(\alpha)
$$

and, by Lemma 3.1(ii), we have $f(\alpha) \geq-f(-\alpha)$, so

$$
\|\alpha\|^{2} \cdot 2 \cdot f(\alpha) / 2 \geq(\alpha,-\alpha) \cdot f(-\alpha) .
$$

By adding these two expressions to $(*)$ and then adding all those short roots with $(\alpha, \beta)=0$, we get

$$
\|\alpha\|^{2} \cdot(k(\alpha)+4) \cdot f(\alpha) / 2 \geq \sum_{\beta \in \Psi}(\alpha, \beta) \cdot f(\beta),
$$

and hence

$$
d(\alpha) \cdot f(\alpha)=(k(\alpha)+4) \cdot f(\alpha) \cdot(\|\alpha\| /\|\sigma\|)^{2} \geq \sum_{\beta \in \Psi} f(\beta) \cdot 2(\alpha, \beta) /\|\beta\|^{2}=\mu(\alpha) .
$$

Again, we have $f(\alpha) \geq \mu(\alpha) / d(\alpha)=\mu(\alpha) / d=\lambda(\alpha)$.

Thus, for both short roots and long roots, we have $f(\alpha) \geq \lambda(\alpha)$ and, since $f(\alpha)$ is an integer, this yields $f(\alpha) \geq\lceil\lambda(\alpha)\rceil=f_{\lambda}(\alpha)$, by Lemma 3.2. Hence since $\mathcal{F}_{\lambda}$ is a Cartan filtration, Lemma 3.1 (iii) yields $\mathcal{F}_{\lambda} \supseteq \mathcal{F}$, and the result follows. Note also that $2(\alpha, \beta) /\|\beta\|^{2} \in \mathbb{Z}$ for all roots $\alpha, \beta \in \Phi$, so $\mu(\Phi) \subseteq \mathbb{Z}$ and $\lambda(\Phi) \subseteq \mathbb{Z} / d$.

The above argument certainly used the fact that $d(\alpha)$ is the same for all roots in $\Phi(L)$. But the proof can also be used to obtain this equality. Indeed, let us write $d_{s}$ for the common value of $d(\alpha)$ over all short roots $\alpha$, guaranteed by the transitivity of the Weyl group, and use $d_{\ell}$ for the case of long roots. Let $\kappa: V \rightarrow \mathbb{R}$ be a nonzero linear functional with $\kappa(\Phi) \subseteq \mathbb{Z}$ and set $\mathcal{F}=\mathcal{F}_{\kappa}$. Then $f(\alpha)=f_{\kappa}(\alpha)=\kappa(\alpha)$ for all $\alpha \in \Phi$ and, as we observed previously, $\mathcal{F}$ is the filtration associated with a $\mathbb{Z}$-grading of $L$.

The latter implies that if $\alpha, \beta$ and $\alpha+\beta$ are in $\Phi$, then $f(\alpha)+f(\beta)=f(\alpha+\beta)$ and $f(\alpha)+f(-\alpha)=0$. In particular, all of the inequalities in the proof of the above lemma are equalities in this special case. It then follows that if $\alpha$ is a long root, then $\kappa(\alpha)=f(\alpha)=\mu(\alpha) / d_{\ell}$. Similarly, if $\alpha$ is a short root, we have $\kappa(\alpha)=f(\alpha)=\mu(\alpha) / d_{s}$. Since the long roots span $V$, by [Hu Lemma 10.4B], the first equation implies that $\kappa=\mu / d_{\ell}$. Since the short roots span $V$, we also have $\kappa=\mu / d_{s}$. In other words, $d_{\ell} \kappa=\mu=d_{s} \kappa$ and, since $\kappa \neq 0$, we conclude that $d_{\ell}=d_{s}$, as required. 


\section{Maximal DUAL Filtrations}

Let $\Phi$ denote the set of roots of the Lie algebra $L$, and let $V$ be the real inner product space they span. In this section, we consider the set $\mathfrak{M}$ of all linear functionals $\lambda$ in the dual space $\widehat{V}$ of $V$ for which the filtration $\mathcal{F}_{\lambda}$ is maximal. We start with a simple observation.

Lemma 4.1. Assume that $\mathcal{F}_{\lambda} \subseteq \mathcal{F}_{\mu}$. If $\alpha \in \Phi$ is a root with $\lambda(\alpha) \in \mathbb{Z}$, then $\lambda(\alpha)=\mu(\alpha)$. In particular, if the root space $V$ has a basis of roots $\left\{\alpha_{1}, \alpha_{2}, \ldots, \alpha_{n}\right\}$ with $\lambda\left(\alpha_{i}\right) \in \mathbb{Z}$ for all $i$, then $\lambda=\mu$ and hence $\mathcal{F}_{\lambda}=\mathcal{F}_{\mu}$.

Proof. Since $\mathcal{F}_{\lambda} \subseteq \mathcal{F}_{\mu}$, it follows from Lemmas 3.1(iii) and 3.2 that the corresponding degree functions satisfy $\lceil\lambda(\omega)\rceil=f_{\lambda}(\omega) \geq f_{\mu}(\omega)=\lceil\mu(\omega)\rceil$ for all roots $\omega \in \Phi$. In particular, when $\omega=\alpha$ or $-\alpha$, we have $\lambda(\alpha) \geq\lceil\mu(\alpha)\rceil \geq \mu(\alpha)$ and $-\lambda(\alpha) \geq\lceil-\mu(\alpha)\rceil \geq-\mu(\alpha)$. Thus $\lambda(\alpha)=\mu(\alpha)$, as required.

Conversely, we have the following key result.

Lemma 4.2. Let $\mathcal{F}_{\lambda}$ be a maximal filtration of $L$. Then the root space $V$ has a basis of roots $\left\{\alpha_{1}, \alpha_{2}, \ldots, \alpha_{n}\right\}$ with $\lambda\left(\alpha_{i}\right) \in \mathbb{Z}$ for all $i$.

Proof. Let $W$ be the real subspace of $V$ spanned by all those roots $\alpha \in \Phi$ with $\lambda(\alpha) \in \mathbb{Z}$. The goal is to show that $W=V$. Suppose, by way of contradiction, that this is not the case. Since $\Phi$ spans $V$, we can extend a basis of $W$ to one of $V$ by adjoining roots $\beta_{0}, \beta_{1}, \ldots, \beta_{k} \in \Phi$ with $k \geq 0$. Let $\delta_{0}$ be the dual basis functional on $V$ corresponding to $\beta_{0}$, so that $\delta_{0}\left(\beta_{0}\right)=1, \delta_{0}\left(\beta_{i}\right)=0$ for $i>0$, and $\delta_{0}(W)=0$. For any real number $t$, we define the functional $\lambda_{t}: V \rightarrow \mathbb{R}$ by $\lambda_{t}=\lambda+t \delta_{0}$, and we study the behavior of this functional with $t$ in the interval $(0,1)$.

Since $\delta_{0}$ vanishes on $W$, we see that $\lambda$ and $\lambda_{t}$ agree on this subspace. Hence $\lambda_{t}$ does not change on roots $\alpha$ with $\lambda(\alpha) \in \mathbb{Z}$. The remaining roots $\omega$ all have $\lambda(\omega)$ strictly between two consecutive integers, and hence $\left\lceil\lambda_{t}(\omega)\right\rceil=\lceil\lambda(\omega)\rceil$ when $t$ is small. On the other hand, $\lambda_{t}\left(\beta_{0}\right)=\lambda\left(\beta_{0}\right)+t$, so $\lambda_{t}\left(\beta_{0}\right)$ will take on an integer value for some $t$ in $(0,1)$. Since the root set $\Phi$ is finite, we can now let $t \in(0,1)$ be minimal with $\lambda_{t}\left(\omega_{0}\right) \in \mathbb{Z}$ for some root $\omega_{0} \notin W$. We consider the associated filtration $\mathcal{F}=\mathcal{F}_{\lambda_{t}}$ with degree function $f=f_{\lambda_{t}}$.

If $\omega \in \Phi \cap W$, then $\lambda_{t}(\omega)=\lambda(\omega)$ and hence, by Lemma 3.2 $f(\omega)=\left\lceil\lambda_{t}(\omega)\right\rceil=$ $\lceil\lambda(\omega)\rceil=f_{\lambda}(\omega)$. If $\omega \in \Phi \backslash W$ with $\lambda_{t}(\omega) \notin \mathbb{Z}$, then the minimality of $t$ implies that $\lambda_{t}(\omega)$ and $\lambda(\omega)$ are in the same open interval $(b, b+1)$ with $b \in \mathbb{Z}$. Hence, we again have $f(\omega)=\left\lceil\lambda_{t}(\omega)\right\rceil=\lceil\lambda(\omega)\rceil=f_{\lambda}(\omega)$. Thus, we need only consider the roots like $\omega_{0}$ with the property that $\lambda_{t}(\omega)$ has just become an integer. Of course, with any such root $\omega$, we also have $\lambda_{t}(-\omega)=-\lambda_{t}(\omega) \in \mathbb{Z}$.

Suppose $\lambda_{t}(\omega)$ has just become an integer and say $a<\lambda(\omega)<a+1$ for some $a \in \mathbb{Z}$. Then $-a-1<\lambda(-\omega)<-a$, so $f_{\lambda}(\omega)=\lceil\lambda(\omega)\rceil=a+1$ and $f_{\lambda}(-\omega)=$ $\lceil\lambda(-\omega)\rceil=-a$. By the minimality of $t$, we know that $\lambda_{t}(\omega)=a$ or $a+1$. If $\lambda_{t}(\omega)=a$, then $\left\lceil\lambda_{t}(\omega)\right\rceil=a$ and $\left\lceil\lambda_{t}(-\omega)\right\rceil=-a$, so

$$
f(\omega)=\left\lceil\lambda_{t}(\omega)\right\rceil=a<a+1=\lceil\lambda(\omega)\rceil=f_{\lambda}(\omega)
$$

and

$$
f(-\omega)=\left\lceil\lambda_{t}(-\omega)\right\rceil=-a=\lceil\lambda(-\omega)\rceil=f_{\lambda}(-\omega) .
$$

On the other hand, if $\lambda_{t}(\omega)=a+1$, then $\left\lceil\lambda_{t}(\omega)\right\rceil=a+1$ and $\left\lceil\lambda_{t}(-\omega)\right\rceil=-a-1$. Thus

$$
f(\omega)=\left\lceil\lambda_{t}(\omega)\right\rceil=a+1=\lceil\lambda(\omega)\rceil=f_{\lambda}(\omega)
$$


and

$$
f(-\omega)=\left\lceil\lambda_{t}(-\omega)\right\rceil=-a-1<-a=\lceil\lambda(-\omega)\rceil=f_{\lambda}(-\omega) .
$$

In either case, we see that the degree function $f$ is strictly smaller than $f_{\lambda}$, so Lemma 3.1(iii) implies that $\mathcal{F}=\mathcal{F}_{\lambda_{t}}$ is strictly larger than $\mathcal{F}_{\lambda}$. Since this contradicts the fact that $\mathcal{F}_{\lambda}$ is a maximal bounded filtration, we conclude that $W=V$.

With this, we can now quickly prove

Proposition 4.3. Let $V$ be the root space of the Lie algebra $L$ and let $\mathcal{F}_{\lambda}$ be a dual filtration with $\lambda: V \rightarrow \mathbb{R}$. Then $\mathcal{F}_{\lambda}$ is a maximal filtration of $L$ if and only if $V$ has a basis $\left\{\alpha_{1}, \alpha_{2}, \ldots, \alpha_{n}\right\}$ consisting of roots with $\lambda\left(\alpha_{i}\right) \in \mathbb{Z}$ for all $i$. Furthermore, when this occurs, then $\mathcal{F}_{\lambda}=\mathcal{F}_{\mu}$ implies that $\lambda=\mu$.

Proof. If $\mathcal{F}_{\lambda}$ is maximal, then the previous lemma implies that a suitable basis $\left\{\alpha_{1}, \alpha_{2}, \ldots, \alpha_{n}\right\} \subseteq \Phi$ exists with $\lambda\left(\alpha_{i}\right) \in \mathbb{Z}$ for all $i$. Conversely, suppose that this basis exists and let $\mathcal{F}$ be any filtration with $\mathcal{F}_{\lambda} \subseteq \mathcal{F}$. Then, by Lemma 3.6, there exists a dual filtration $\mathcal{F}_{\mu}$ with $\mathcal{F} \subseteq \mathcal{F}_{\mu}$. Since $\mathcal{F}_{\lambda} \subseteq \mathcal{F}_{\mu}$, Lemma 4.1 and the basis information now imply that $\lambda=\mu$, so $\mathcal{F}_{\lambda}=\mathcal{F}=\mathcal{F}_{\mu}$ and we conclude that $\mathcal{F}_{\lambda}$ is indeed maximal. A second application of Lemma 4.1 yields the result.

For each of the finitely many bases $\mathfrak{B}$ of $V$ consisting of roots, let

$$
\Lambda_{\mathfrak{B}}=\{\lambda \in \widehat{V} \mid \lambda(\mathfrak{B}) \subseteq \mathbb{Z}\} .
$$

Since $\operatorname{dim}_{\mathbb{R}} V=n$, it follows that $\Lambda_{\mathfrak{B}}$ is a subgroup of $\widehat{V}$ isomorphic to $\mathbb{Z}^{n}$. Now suppose that $\mathfrak{B}_{0} \subseteq \Phi$ is a basis of $\Phi$. By this, we mean that any root in $\Phi$ is an integer linear combination of the elements of $\mathfrak{B}_{0}$, and uniquely so. Setting

$$
\Lambda_{\Phi}=\{\lambda \in \widehat{V} \mid \lambda(\Phi) \subseteq \mathbb{Z}\},
$$

we see that $\Lambda_{\Phi}=\Lambda_{\mathfrak{B}_{0}}, \Lambda_{\mathfrak{B}} \supseteq \Lambda_{\Phi}$ and $\left|\Lambda_{\mathfrak{B}}: \Lambda_{\Phi}\right|<\infty$ for any basis $\mathfrak{B} \subseteq \Phi$ of $V$. Finally, if $d$ is the common value of the parameter $d(\alpha)$ as given by Lemma 3.4. then clearly

$$
\Lambda_{\Phi} / d=\{\lambda \in \widehat{V} \mid \lambda(\Phi) \subseteq \mathbb{Z} / d\} .
$$

With all this notation, we have

Corollary 4.4. Let $\mathfrak{M} \subseteq \widehat{V}$ be the set of all linear functionals $\lambda$ with $\mathcal{F}_{\lambda}$ a maximal bounded filtration.

(i) $\mathfrak{M}=\bigcup_{\mathfrak{B}} \Lambda_{\mathfrak{B}}$, a finite union of subgroups of $\widehat{V}$, each isomorphic to $\mathbb{Z}^{n}$.

(ii) If $\lambda \in \mathfrak{M}$, then $\mathbb{Z} \lambda \subseteq \mathfrak{M}$.

(iii) $\mathfrak{M}$ is a finite union of cosets of the group $\Lambda_{\Phi} \cong \mathbb{Z}^{n}$.

(iv) $\mathfrak{M} \subseteq \Lambda_{\Phi} / d$.

Proof. Part (i) is an immediate consequence of Proposition 4.3. and parts (ii) and (iii) follow directly from it. Finally, if $\lambda \in \mathfrak{M}$ then, by Lemma 3.6, there exists a functional $\lambda^{\prime} \in \Lambda_{\Phi} / d$ with $\mathcal{F}_{\lambda} \subseteq \mathcal{F}_{\lambda^{\prime}}$. Thus $\mathcal{F}_{\lambda}=\mathcal{F}_{\lambda^{\prime}}$ and hence $\lambda=\lambda^{\prime} \in \Lambda_{\Phi} / d$ by the uniqueness aspect of Proposition 4.3 .

In the next two sections, we will take a closer look at $\mathfrak{M}$ for each type of simple Lie algebra. 


\section{Maximal dual filtrations for $A_{n}, B_{n}, C_{n}, D_{n}$}

In view of Lemma 3.6, the maximal Cartan filtrations of $L$ are necessarily dual filtrations associated to linear functionals $\lambda: V \rightarrow \mathbb{R}$. In this section, we determine precisely which dual filtrations are maximal for the families $A_{n}, B_{n}, C_{n}$ and $D_{n}$. Surprisingly, there are real differences between these four cases, and consequently it is necessary to deal with them separately. We start with $A_{n}$, where the filtrations behave quite like those of full matrix rings (see $[\mathrm{P}$, Theorem 3.6(ii)]).

Proposition 5.1. Let $L$ be a simple Lie algebra of type $A_{n}$, with $n \geq 1$, and let $\mathcal{F}$ be a bounded Cartan $\mathbb{Z}$-filtration of $L$. Then $\mathcal{F}$ is maximal if and only if $\mathcal{F}=\mathcal{F}_{\lambda}$, where $\lambda: V \rightarrow \mathbb{R}$ is a linear functional with $\lambda(\Phi) \subseteq \mathbb{Z}$.

Proof. If $\mathcal{F}=\mathcal{F}_{\lambda}$ with $\lambda(\Phi) \subseteq \mathbb{Z}$, then it follows immediately from Proposition 4.3 that $\mathcal{F}$ is maximal.

Conversely, suppose $\mathcal{F}$ is a maximal Cartan filtration. Then Lemma 3.6 implies that $\mathcal{F}=\mathcal{F}_{\lambda}$ for some functional $\lambda$. Let $W$ be the real inner product space of dimension $n+1$ with orthonormal basis $\left\{e_{0}, e_{1}, \ldots, e_{n}\right\}$. Then $V$ is a hyperplane in $W$ and $\Phi(L)$ is the set of all $e_{i}-e_{j}$ with $i \neq j$. Extend $\lambda$ to a linear functional on $W$ and let $\lambda\left(e_{i}\right)=w_{i} \in \mathbb{R}$. Set $v_{i}=\left\lceil w_{i}\right\rceil$ and define $\mu: W \rightarrow \mathbb{R}$ by $\mu\left(e_{i}\right)=v_{i}$. If $f_{\lambda}$ is the degree function of $\mathcal{F}_{\lambda}$ and if $f_{\mu}$ is the degree function for $\mathcal{F}_{\mu}$, then for all roots $\alpha=e_{i}-e_{j}$ we have

$$
f_{\lambda}(\alpha)=\lceil\lambda(\alpha)\rceil=\left\lceil w_{i}-w_{j}\right\rceil \geq\left\lceil w_{i}\right\rceil-\left\lceil w_{j}\right\rceil=v_{i}-v_{j}=\mu(\alpha)=f_{\mu}(\alpha),
$$

by $\left[\mathrm{P}\right.$, Lemma 3.3]. Thus Lemma 3.1(iii) implies that $\mathcal{F}_{\lambda} \subseteq \mathcal{F}_{\mu}$ and, by the maximality of $\mathcal{F}_{\lambda}$, we have $\mathcal{F}_{\lambda}=\mathcal{F}_{\mu}$. Hence, the uniqueness aspect of Proposition 4.3 yields $\lambda=\mu$, and therefore $\lambda(\Phi)=\mu(\Phi) \subseteq \mathbb{Z}$.

For the remaining three families we consider in this section, it is convenient to first isolate a few arithmetic facts. For any real number $x$, let us define its average to be

$$
\operatorname{av} x=\frac{\lfloor x\rfloor+\lceil x\rceil}{2} .
$$

Note that av $x \in \mathbb{Z} / 2$, the set of half integers. Indeed, if $x$ is an integer, then certainly av $x=x$. On the other hand, if $k<x<k+1$ for some $k \in \mathbb{Z}$, then av $x=k+(1 / 2)$. When we speak about the parity of a number in $\mathbb{Z} / 2$, we will mean the parity of its numerator. Thus, the even parity numbers are precisely the integers, while the odd parity numbers are elements of the set $\mathbb{Z}+(1 / 2)$.

Lemma 5.2. Let $x, y \in \mathbb{R}$. Then we have $\lceil x\rceil \geq \operatorname{av} x, \operatorname{av}(-x)=-(\operatorname{av} x)$, and $\lceil x+y\rceil \geq$ av $x+$ av $y$.

Proof. If $x$ is an integer, then $\operatorname{av}(-x)=-x=-(\operatorname{av} x)$. On the other hand, if $k<$ $x<k+1$ for some $k \in \mathbb{Z}$, then av $x=k+(1 / 2)$. Furthermore, $-k-1<-x<-k$, so $\operatorname{av}(-x)=-k-1+(1 / 2)=-k-(1 / 2)=-(\operatorname{av} x)$.

Now, if $y$ is an integer, then

$$
\lceil x+y\rceil=\lceil x\rceil+y \geq \operatorname{av} x+\operatorname{av} y .
$$

Similarly, the inequality holds if $x$ is an integer. Finally, if $a<x<a+1$ and $b<y<b+1$ with $a, b \in \mathbb{Z}$, then $x+y>a+b$, so

$$
\lceil x+y\rceil \geq a+b+1=(a+(1 / 2))+(b+(1 / 2))=\operatorname{av} x+\operatorname{av} y,
$$

as required. 
For the simple Lie algebras of type $B_{n}, C_{n}$ or $D_{n}$, it seems more appropriate to describe the functionals in terms of the orthonormal basis $\Omega=\left\{e_{1}, e_{2}, \ldots, e_{n}\right\}$ for $V$ given by [B, Plates II-IV]. Note however that we allow $C_{2}$ to occur here, but we insist that $n \geq 3$ for $B_{n}$ and $D_{n}$.

Lemma 5.3. Let $L$ be a simple Lie algebra of type $B_{n}, C_{n}$ or $D_{n}$. If $\lambda: V \rightarrow \mathbb{R}$ is a linear functional, then there exists a linear functional $\mu: V \rightarrow \mathbb{R}$ with $\mu(\Omega) \subseteq \mathbb{Z} / 2$ and with $\mathcal{F}_{\lambda} \subseteq \mathcal{F}_{\mu}$.

Proof. Let $\lambda\left(e_{i}\right)=w_{i}$ and define $\mu: V \rightarrow \mathbb{R}$ by $\mu\left(e_{i}\right)=\operatorname{av}\left(w_{i}\right)$ for $i=1,2, \ldots, n$. Then certainly $\mu(\Omega) \subseteq \mathbb{Z} / 2$ and we claim that $\mathcal{F}_{\lambda} \subseteq \mathcal{F}_{\mu}$. In view of Lemmas 3.1(iii) and 3.2 it suffices to show that $\lceil\lambda(\alpha)\rceil=f_{\lambda}(\alpha) \geq f_{\mu}(\alpha)=\lceil\mu(\alpha)\rceil$ for all roots $\alpha \in \Phi$. To start with, consider the roots $\alpha= \pm e_{i} \pm e_{j}$, possibly with $i=j$. Then, for any fixed choice of signs, Lemma 5.2 implies that

$$
\lceil\lambda(\alpha)\rceil=\left\lceil \pm w_{i} \pm w_{j}\right\rceil \geq \operatorname{av}\left( \pm w_{i}\right)+\operatorname{av}\left( \pm w_{j}\right)= \pm \operatorname{av}\left(w_{i}\right) \pm \operatorname{av}\left(w_{j}\right)=\mu(\alpha),
$$

so $\lceil\lambda(\alpha)\rceil \geq\lceil\mu(\alpha)\rceil$. On the other hand, if $\alpha= \pm e_{i}$, the same argument applies by merely setting $w_{j}=0$.

Finally, we combine the proofs of the following three key results.

Proposition 5.4. Let $L$ be a simple Lie algebra of type $B_{n}$, with $n \geq 3$, and let $\mathcal{F}$ be a bounded Cartan $\mathbb{Z}$-filtration of L. Then $\mathcal{F}$ is maximal if and only if $\mathcal{F}=\mathcal{F}_{\lambda}$, where $\lambda: V \rightarrow \mathbb{R}$ is a linear functional with $\lambda(\Omega) \subseteq \mathbb{Z} / 2$ satisfying the additional property that there is no subscript $i_{0}$ such that $\lambda\left(e_{i_{0}}\right) \in \mathbb{Z}+(1 / 2)$ while $\lambda\left(e_{i}\right) \in \mathbb{Z}$ for the remaining $i \neq i_{0}$.

Since the short roots of Lie algebras of type $B_{n}$ are all of the form $\pm e_{i}$, the condition $\lambda(\Omega) \subseteq \mathbb{Z} / 2$ can be replaced by the assertion that $\lambda$ takes on half integer values on the short roots.

Proposition 5.5. Let $L$ be a simple Lie algebra of type $C_{n}$, with $n \geq 2$, and let $\mathcal{F}$ be a bounded Cartan $\mathbb{Z}$-filtration of $L$. Then $\mathcal{F}$ is maximal if and only if $\mathcal{F}=\mathcal{F}_{\lambda}$, where $\lambda: V \rightarrow \mathbb{R}$ is a linear functional with $\lambda(\Omega) \subseteq \mathbb{Z} / 2$.

Since the long roots of $L$ above are of the form $\pm 2 e_{i}$, the condition $\lambda(\Omega) \subseteq \mathbb{Z} / 2$ is equivalent to the assertion that $\lambda$ takes on integer values on the long roots. If we choose any such $\lambda$ so that not all $\lambda\left(e_{i}\right)$ have the same parity, then some $\lambda\left(e_{i}\right)+\lambda\left(e_{j}\right)$ is not an integer. In particular, $\lambda(\Phi) \nsubseteq \mathbb{Z}$, and hence we obtain a maximal Cartan filtration $\mathcal{F}_{\lambda}$ that is not associated to a $\mathbb{Z}$-grading.

Proposition 5.6. Let $L$ be a simple Lie algebra of type $D_{n}$, with $n \geq 3$, and let $\mathcal{F}$ be a bounded Cartan $\mathbb{Z}$-filtration of $L$. Then $\mathcal{F}$ is maximal if and only if $\mathcal{F}=\mathcal{F}_{\lambda}$, where $\lambda: V \rightarrow \mathbb{R}$ is a linear functional with $\lambda(\Omega) \subseteq \mathbb{Z} / 2$ satisfying the additional property that there is no subscript $i_{0}$ such that $\lambda\left(e_{i_{0}}\right)$ has a parity different from that of the remaining $\lambda\left(e_{i}\right)$.

Proof. By Lemma 3.6, we know that any maximal Cartan filtration is necessarily a dual filtration $\mathcal{F}_{\lambda}$ and, by Lemma 5.3 and the uniqueness part of Proposition 4.3 , we know that $\lambda(\Omega) \subseteq \mathbb{Z} / 2$. Thus, by Propostion 4.3 again, we need only determine those $\lambda \in \widehat{V}$ with $\lambda(\Omega) \subseteq \mathbb{Z} / 2$ and with $\lambda(\mathfrak{B}) \subseteq \mathbb{Z}$ for some basis $\mathfrak{B}$ of $V$ consisting entirely of roots. 
To start with, if $L$ is of type $C_{n}$, then $\mathfrak{B}=\left\{2 e_{1}, 2 e_{2}, \ldots, 2 e_{n}\right\} \subseteq \Phi$. Furthermore, $\mathfrak{B}$ is clearly a basis for $V$ and $\lambda(\mathfrak{B}) \subseteq \mathbb{Z}$, so any such $\lambda$ yields a maximal filtration. This proves Proposition 5.5 .

Now let $L$ be of type $B_{n}$ or $D_{n}$, with $n \geq 3$, and let $\lambda$ be given. For convenience, write $\Omega_{+}=\left\{e_{i} \mid \lambda\left(e_{i}\right) \in \mathbb{Z}\right\}$ and $\Omega_{-}=\left\{e_{i} \mid \lambda\left(e_{i}\right) \in \mathbb{Z}+(1 / 2)\right\}$. If $i \neq j$, and $\lambda\left(e_{i}\right)$ and $\lambda\left(e_{j}\right)$ have the same parity, then $\lambda\left(e_{i}+e_{j}\right)$ and $\lambda\left(e_{i}-e_{j}\right)$ both belong to $\mathbb{Z}$. Furthermore, $e_{i}+e_{j}$ and $e_{i}-e_{j}$ are roots, and together they span $\mathbb{R} e_{i}+\mathbb{R} e_{j}$. With this, it follows easily that if $\left|\Omega_{+}\right| \neq 1$, then $\mathbb{R} \Omega_{+}$has a basis $\mathfrak{B}_{+}$of roots with $\lambda\left(\mathfrak{B}_{+}\right) \subseteq \mathbb{Z}$. Similarly, if $\left|\Omega_{-}\right| \neq 1$, then $\mathbb{R} \Omega_{-}$has a basis $\mathfrak{B}_{-}$of roots with $\lambda\left(\mathfrak{B}_{-}\right) \subseteq \mathbb{Z}$. In particular, since $\mathbb{R} \Omega_{+}+\mathbb{R} \Omega_{-}=\mathbb{R} \Omega=V$, we conclude that $\mathcal{F}_{\lambda}$ is maximal unless $\left|\Omega_{+}\right|=1$ or $\left|\Omega_{-}\right|=1$.

Since $|\Omega|=n \geq 3$, it therefore suffices to assume that $\left|\Omega_{+}\right|=1$ or $\left|\Omega_{-}\right|=1$, but not both. In other words, there exists a unique subscript $i_{0}$ such that $\lambda\left(e_{i_{0}}\right)$ has parity different from the remaining $\lambda\left(e_{i}\right)$. If $L$ is of type $D_{n}$, then the roots of $L$ are all of the form $\pm e_{i} \pm e_{j}$, with $i \neq j$, and it is clear that $e_{i_{0}}$ cannot belong to the space spanned by the roots $\alpha$ with $\lambda(\alpha) \in \mathbb{Z}$. Thus, in this case, $\mathcal{F}_{\lambda}$ is not maximal, and consequently Proposition 5.6 is proved.

On the other hand, if $L$ is of type $B_{n}$, then $L$ has the additional roots $\pm e_{i}$. In particular, if $\lambda\left(e_{i_{0}}\right) \in \mathbb{Z}$, then there does exist a suitable basis of roots $\alpha$ with $\lambda(\alpha) \in \mathbb{Z}$. Thus, the only exceptional case here occurs when $\lambda\left(e_{i_{0}}\right) \in \mathbb{Z}+(1 / 2)$. This completes the proof.

One can prove directly that the exceptional filtrations in both Propositions 5.4 and 5.6 are not maximal. Indeed, let $\lambda \in \widehat{V}$ and $e_{i_{0}} \in \Omega$ be given with $\lambda\left(e_{i_{0}}\right)$ having parity different from the remaining $\lambda\left(e_{i}\right)$, and set $\Omega^{\prime}=\Omega \backslash\left\{e_{i_{0}}\right\}$. If $\mu: V \rightarrow \mathbb{R}$ agrees with $\lambda$ on $\mathbb{R} \Omega^{\prime}$ and if $\mu\left(e_{i_{0}}\right)=\lambda\left(e_{i_{0}}\right) \pm(1 / 2)$, then the argument of Lemma 4.2, applied to any root $\omega=e_{i_{0}}+e_{j}$, shows that $\mathcal{F}_{\lambda}$ is strictly smaller than $\mathcal{F}_{\mu}$.

\section{Maximal dual filtrations for $E_{6}, E_{7}, E_{8}, F_{4}$ And $G_{2}$}

Again, we let $L$ denote a finite-dimensional simple complex Lie algebra with root set $\Phi$ and with root space $V$ of dimension $n$. Recall that $\mathfrak{M}$ is the subset of $\widehat{V}$ consisting of all linear functionals $\lambda$ such that $\mathcal{F}_{\lambda}$ is a maximal filtration of $L$, and that $\Lambda_{\Phi} \cong \mathbb{Z}^{n}$ is the subgroup of $\widehat{V}$ consisting of all linear functionals $\lambda$ with $\lambda(\Phi) \subseteq \mathbb{Z}$. Furthermore, if $d$ is the common value of the parameters $d(\alpha)$ as given by Lemma 3.4, then Corollary 4.4(iii), (iv) asserts that $\mathfrak{M}$ is a finite union of cosets of $\Lambda_{\Phi}$ and that

$$
\Lambda_{\Phi} \subseteq \mathfrak{M} \subseteq \Lambda_{\Phi} / d=\left\{\lambda \in \widehat{V} \mid d \lambda \in \Lambda_{\Phi}\right\} \cong \mathbb{Z}^{n} .
$$

It follows that there exists a smallest positive integer $e=e(L)$ with $e \lambda \in \Lambda_{\Phi}$ for all $\lambda \in \mathfrak{M}$, and that $e$ divides $d$. In particular, $d$ is an upper bound for $e$.

We can also obtain an easy lower bound for the parameter $e$. To this end, we fix a basis $\mathfrak{B}_{0}=\left\{\beta_{1}, \beta_{2}, \ldots, \beta_{n}\right\} \subseteq \Phi$ for $\Phi$. By this we mean that $\mathfrak{B}_{0}$ is a basis for $V$ and that every root in $\Phi$ is an integral linear combination of the members of $\mathfrak{B}_{0}$. In particular, if $\alpha \in \Phi$, then we can write $\alpha=\sum_{i=1}^{n} c_{i} \beta_{i}$ uniquely with $c_{i} \in \mathbb{Z}$, and we define $f(\alpha)$ to be the (positive) least common multiple of the nonzero coefficients $c_{i}$. With this notation, we have

Lemma 6.1. If $\alpha \in \Phi$, then $f(\alpha)$ divides $e=e(L)$. 
Proof. Write $\alpha=c_{1} \beta_{1}+c_{2} \beta_{2}+\cdots+c_{n} \beta_{n}$ and, for convenience, suppose that $c_{n} \neq 0$. If $\lambda: V \rightarrow \mathbb{R}$ is defined by $\lambda\left(\beta_{j}\right)=0$ for all $j \neq n$ and $\lambda\left(\beta_{n}\right)=1 / c_{n}$, then $\lambda$ takes on integer values on the set $\left\{\beta_{1}, \beta_{2}, \ldots, \beta_{n-1}, \alpha\right\}$ which is clearly an $\mathbb{R}$-basis of $V$ consisting of roots. Thus, by Proposition 4.3 , we see that $\lambda \in \mathfrak{M}$. Furthermore, $\left|c_{n}\right|$ is clearly the smallest positive integer $t$ with $t \lambda \in \Lambda_{\Phi}$. Thus $\left|c_{n}\right|$ divides $e$, and hence $f(\alpha)$ divides $e$.

One expects the above result to yield the most information when the coefficients $c_{i}$ are large, and this is indeed the case. Specifically, we use the bases given in the nine plates at the end of $[\mathrm{BO}$, and we note that these plates also contain information on the coefficients of all positive roots having at least one coefficient larger than 1 . With this, it is easy to verify that each $f(\alpha)$ divides $f=f(\beta)$, where $\beta$ is a highest root of $L$. The $f$-row of the following chart is obtained from this data.

\begin{tabular}{||c||c|c|c|c|c|c|c|c|c||}
\hline \hline Type & $A_{n}$ & $B_{n}$ & $C_{n}$ & $D_{n}$ & $E_{6}$ & $E_{7}$ & $E_{8}$ & $F_{4}$ & $G_{2}$ \\
\hline \hline$d$ & $2(n+1)$ & 4 & $4(n-1)$ & $4(n-1)$ & 24 & 36 & 60 & 12 & 6 \\
$e$ & 1 & 2 & 2 & 2 & $?$ & $?$ & 60 & 12 & 6 \\
$f$ & 1 & 2 & 2 & 2 & 6 & 12 & 60 & 12 & 6 \\
\hline \hline
\end{tabular}

Of course, the $d$-row in the above comes from the chart immediately preceding Lemma 3.4, while the $e$-entries for $A_{n}, B_{n}, C_{n}$ and $D_{n}$ come from the work of Section 5. Finally, the $e$-entries for $E_{8}, F_{4}$ and $G_{2}$ follow from the facts that $f$ divides $e$ and that $e$ divides $d$. This leaves only two unknown values, namely $e\left(E_{6}\right)$ which is either 6,12 or 24 , and $e\left(E_{7}\right)$ which is either 12 or 24 . It is interesting to note that $e=f$ for all of the known values.

In the remainder of this section, we will describe specific computations, using Maple 9, for the algebras $G_{2}$ and $F_{4}$. For the five exceptional Lie algebras, computations can be based on the fact that $\mathfrak{M} \subseteq \Lambda_{\Phi} / d$ and that $\mathfrak{M}$ consists of a finite union of cosets of the subgroup $\Lambda_{\Phi}$. Indeed, since $\left|\Lambda_{\Phi} / d: \Lambda_{\Phi}\right|=d^{n}$, this means that we need only test $d^{n}$ coset representatives and determine which of these elements are in $\mathfrak{M}$. Furthermore, it is clear that these coset representatives can be easily described in terms of a basis $\mathfrak{B}_{0}=\left\{\beta_{1}, \beta_{2}, \ldots, \beta_{n}\right\} \subseteq \Phi$ of $\Phi$. In fact, one posssible choice for this set is given by all those $\lambda \in \widehat{V}$ such that $\lambda\left(\beta_{i}\right)=0 / d, 1 / d, \ldots$, or $(d-1) / d$ for all $i=1,2, \ldots, n$. Finally, using Proposition 4.3, we can test whether each such $\lambda$ is in $\mathfrak{M}$ by determining all roots $\alpha \in \Phi$ with $\lambda(\alpha) \in \mathbb{Z}$ and seeing whether there are enough of these roots to span the vector space $V$.

The general approach is as follows. Let $A$ denote the matrix whose rows are indexed by the positive roots $\alpha \in \Phi$ and whose entries in the $\alpha$-row are the coefficients in the expression for $\alpha$ as an integral linear combination of the basis $\mathfrak{B}_{0}$. For example, if $L$ is of type $G_{2}$, then, as in [Bo, Plate IX], we have $\mathfrak{B}_{0}=\left\{\beta_{1}, \beta_{2}\right\}$ and positive roots $\beta_{1}, \beta_{2}, \beta_{1}+\beta_{2}, 2 \beta_{1}+\beta_{2}, 3 \beta_{1}+\beta_{2}$, and $3 \beta_{1}+2 \beta_{2}$. In particular, in this case, $A$ is the $6 \times 2$ matrix whose transpose $A^{T}$ is given by

$$
A^{T}=\left[\begin{array}{llllll}
1 & 0 & 1 & 2 & 3 & 3 \\
0 & 1 & 1 & 1 & 1 & 2
\end{array}\right] .
$$

Next, for each of the $d^{n}$ choices of $\lambda$ we form the column matrix $B$ whose entries are $\lambda\left(\beta_{1}\right), \lambda\left(\beta_{2}\right), \ldots, \lambda\left(\beta_{n}\right)$. Then $A B$ is a column matrix with $\alpha$-row entry precisely equal to $\lambda(\alpha)$. Thus, we need only determine which of these entries are integers, 
obtain the submatrix $C$ of $A$ consisting of all those $\alpha$-rows with $\lambda(\alpha) \in \mathbb{Z}$, and then determine the rank of $C$. By Proposition 4.3, $\lambda \in \mathfrak{M}$ if and only if rank $C=n$.

Of course, as a practical matter, we merely keep track of the numerators of each $\lambda\left(\beta_{i}\right)$ and work in the ring $\mathbb{Z} / d \mathbb{Z}$. Indeed, by so doing, we can actually think of these coset representatives as being members of the factor group $\left(\Lambda_{\Phi} / d\right) /\left(\Lambda_{\Phi}\right)$ which is isomorphic to $(\mathbb{Z} / d \mathbb{Z})^{n}$.

When $L$ is of type $G_{2}$, there are $d^{n}=6^{2}=36$ possibilities to consider and 6 of these (or $16.7 \%$ ) turn out to be members of $\mathfrak{M}$. These are listed in the chart below, where each entry is an ordered pair of numbers in $\mathbb{Z} / 6 \mathbb{Z}$ corresponding to the values $\left(\lambda\left(\beta_{1}\right), \lambda\left(\beta_{2}\right)\right)$.

\begin{tabular}{|c|c|c|c|c|c|}
\hline \multicolumn{4}{|c|}{$G_{2}:$ Order $\leq 2$} & \multicolumn{2}{|c|}{ 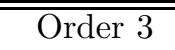 } \\
\hline$\overline{(0,0)}$ & $(0,3)$ & $(3,0)$ & $(3,3)$ & $(2,0)$ & $(4,0$ \\
\hline
\end{tabular}

As we remarked above, we view these elements as members of the factor group $\left(\Lambda_{\Phi} / 6\right) /\left(\Lambda_{\Phi}\right) \cong(\mathbb{Z} / 6 \mathbb{Z})^{2}$. Since the first four of these elements have order $\leq 2$, we see that they constitute all four elements of $(\mathbb{Z} / 2 \mathbb{Z})^{2} \subseteq(\mathbb{Z} / 6 \mathbb{Z})^{2}$. Translating this back to $\Lambda_{\Phi} / 6$, it follows that $\mathfrak{M} \supseteq \Lambda_{\Phi} / 2$. The remaining two elements have order 3 and are inverses of each other. We conclude that $\mathfrak{M}=\left(\Lambda_{\Phi} / 2\right) \cup H$, where $H$ is the cyclic extension of $\Lambda_{\Phi}$ of type (3) generated by the functional $\lambda$ with $\lambda\left(\beta_{1}\right)=1 / 3$ and $\lambda\left(\beta_{2}\right)=0$. This, of course, agrees with the result of Corollary 4.4(i), which asserts that $\mathfrak{M}$ is a finite union of subgroups of $\widehat{V}$, each containing $\Lambda_{\Phi}$.

The computations for $L$ of type $F_{4}$ proceed in a similar manner. In this case, it is easier to describe the roots in terms of the elements $\left\{e_{1}, e_{2}, e_{3}, e_{4}\right\}$, and so the matrix $A$ is constructed using this basis for $V$, and its entries are integers and half integers. For any coset representative $\lambda$ defined by its values on $\mathfrak{B}_{0}=$ $\left\{e_{2}-e_{3}, e_{3}-e_{4}, e_{4},\left(e_{1}-e_{2}-e_{3}-e_{4}\right) / 2\right\}$, we first compute $\lambda\left(e_{1}\right), \lambda\left(e_{2}\right), \lambda\left(e_{3}\right), \lambda\left(e_{4}\right)$ and use these as the entries of the column matrix $B$. Again, the $\alpha$-entry of the column matrix $A B$ is equal to $\lambda(\alpha)$. There are $12^{4}=20,736$ possibilities to consider and surprisingly only 72 (or $0.35 \%$ ) correspond to members of $\mathfrak{M}$. These 72 coset representatives, viewed in $(\mathbb{Z} / 12 \mathbb{Z})^{4}$, are listed in the following three tables. The first table indicates that the coset representatives in $\mathfrak{M}$, viewed in the factor group, include all 16 elements of $(\mathbb{Z} / 12 \mathbb{Z})^{4}$ having order $\leq 2$, and hence $\mathfrak{M} \supseteq \Lambda_{\Phi} / 2$.

\begin{tabular}{||c|c|c|c||}
\hline \hline \multicolumn{4}{|c||}{$F_{4}:$ Order $\leq 2$} \\
\hline$(0,0,0,0)$ & $(6,0,0,0)$ & $(0,6,0,0)$ & $(0,0,6,0)$ \\
$(0,0,0,6)$ & $(6,6,0,0)$ & $(6,0,6,0)$ & $(6,0,0,6)$ \\
$(0,6,6,0)$ & $(0,6,0,6)$ & $(0,0,6,6)$ & $(6,6,6,0)$ \\
$(6,6,0,6)$ & $(6,0,6,6)$ & $(0,6,6,6)$ & $(6,6,6,6)$ \\
\hline \hline
\end{tabular}

Next, we consider the set $\mathfrak{S}_{4}$ of coset representatives in $\mathfrak{M}$ having order 4 modulo $\Lambda_{\Phi}$. There are 24 of these and surprisingly they group into three sets of size 8 each having the same square. Thus the elements in the first two rows each have square $(0,0,0,6)$, the elements in the third and fourth rows have square $(0,0,6,0)$, and those in the last two rows have square $(0,0,6,6)$. Note that, in each pair of rows, each element occurs with its inverse in the same column. 


\begin{tabular}{||c|c|c|c||}
\hline \hline \multicolumn{4}{|c||}{$F_{4}:$ Order 4} \\
\hline$(0,0,6,3)$ & $(0,6,0,3)$ & $(6,0,6,3)$ & $(6,6,6,3)$ \\
$(0,0,6,9)$ & $(0,6,0,9)$ & $(6,0,6,9)$ & $(6,6,6,9)$ \\
\hline$(0,0,3,0)$ & $(0,6,3,6)$ & $(6,0,3,6)$ & $(6,6,3,6)$ \\
$(0,0,9,0)$ & $(0,6,9,6)$ & $(6,0,9,6)$ & $(6,6,9,6)$ \\
\hline$(0,0,3,3)$ & $(0,6,3,9)$ & $(6,0,3,3)$ & $(6,6,3,3)$ \\
$(0,0,9,9)$ & $(0,6,9,3)$ & $(6,0,9,9)$ & $(6,6,9,9)$ \\
\hline \hline
\end{tabular}

As in our previous discussion, we would like to combine as many of these elements as possible into subgroups of $(\mathbb{Z} / 12 \mathbb{Z})^{4}$ consisting entirely of elements of $\mathfrak{M} / \Lambda_{\Phi}$. To start with, if $x, y \in \mathfrak{S}_{4}$ have different squares, then it is easy to check that $x y^{2} \notin \mathfrak{M}$. Thus, we can only group together elements with the same square. In this case, it is tempting to suspect, for example, that the 8 elements in the first two rows are the eight elements of order 4 in a group isomorphic to $(\mathbb{Z} / 4 \mathbb{Z}) \times(\mathbb{Z} / 2 \mathbb{Z})^{2}$, but it is easy to check that this is not the case, Thus, the best we can hope to do is to combine elements into groups of order 8 , and this is quite easy. Indeed, let $x$ and $y$ be commuting group elements of order 4 with $x^{2}=y^{2}$ and $y \neq x^{ \pm 1}$. Then $x$ and $y$ generate a group $\langle x, y\rangle$ of order 8 having $x^{ \pm 1}, y^{ \pm 1}$ as its four elements of order 4 . Since we know that all elements of order 1 and 2 are contained in $\mathfrak{M} / \Lambda_{\Phi}$, it follows that $\langle x, y\rangle \subseteq \mathfrak{M} / \Lambda_{\Phi}$. In particular, by combining elements of order 4 as above, we can partition $\mathfrak{S}_{4}$, in numerous ways, as the disjoint union of six sets, each corresponding to the elements of order 4 in a subgroup of $(\mathbb{Z} / 12 \mathbb{Z})^{4}$ isomorphic to $(\mathbb{Z} / 4 \mathbb{Z}) \times(\mathbb{Z} / 2 \mathbb{Z})$. Hence $\mathfrak{M} \supseteq \bigcup_{i=1}^{6} H_{i}$, where each $H_{i}$ is a subgroup of $\widehat{V}$ which is a $(4,2)$ extension of $\Lambda_{\Phi}$.

Finally, $\mathfrak{M} / \Lambda_{\Phi}$ contains 32 elements of order 3 , and we denote the subset of these elements by $\mathfrak{S}_{3}$. Furthermore, it is not hard to see that $\left(\Lambda_{\Phi} / 12\right) /\left(\Lambda_{\Phi}\right)$ contains precisely eight subgroups of type $(3,3)$ having their 8 nonidentity elements in $\mathfrak{S}_{3}$, and each element of $\mathfrak{S}_{3}$ is contained in precisely two of these subgroups. Since any such subgroup intersects four others nontrivially, it is necessarily disjoint from the remaining three. As a consequence, if $\mathfrak{S}_{3}$ can be partitioned into a disjoint union of some of these 8 element sets, then each of these subsets can be contained in at most one partition of this type. In particular, $\mathfrak{S}_{3}$ has at most two such partitions, one of which is given below. Here, each pair of rows constitutes an 8 element set, and each element occurs with its inverse in the same column.

\begin{tabular}{||c|c|c|c||}
\hline \hline \multicolumn{5}{||c||}{$F_{4}$ : Order 3 (Partition 1) } \\
\hline$(0,0,4,0)$ & $(4,4,0,4)$ & $(4,4,4,4)$ & $(4,4,8,4)$ \\
$(0,0,8,0)$ & $(8,8,0,8)$ & $(8,8,8,8)$ & $(8,8,4,8)$ \\
\hline$(0,4,0,0)$ & $(4,0,8,8)$ & $(4,4,8,8)$ & $(4,8,8,8)$ \\
$(0,8,0,0)$ & $(8,0,4,4)$ & $(8,8,4,4)$ & $(8,4,4,4)$ \\
\hline$(0,4,0,4)$ & $(4,0,4,4)$ & $(4,4,4,8)$ & $(4,8,4,0)$ \\
$(0,8,0,8)$ & $(8,0,8,8)$ & $(8,8,8,4)$ & $(8,4,8,0)$ \\
\hline$(0,4,4,8)$ & $(4,0,8,0)$ & $(4,4,0,8)$ & $(4,8,4,4)$ \\
$(0,8,8,4)$ & $(8,0,4,0)$ & $(8,8,0,4)$ & $(8,4,8,8)$ \\
\hline \hline
\end{tabular}

As a consequence, the above table describes four of the eight $(3,3)$ subgroups. As it turns out, a second partition also exists and is given below. Hence, the following 
table describes the remaining four subgroups. By using either of these partitions, along with our previous comments on the elements of order 2 and 4, we conclude that $\mathfrak{M}$ can be described as a union of eleven subgroups, namely

$$
\mathfrak{M}=\left(\Lambda_{\Phi} / 2\right) \cup \bigcup_{i=1}^{6} H_{i} \cup \bigcup_{j=1}^{4} \widetilde{H}_{j}
$$

where each $\widetilde{H}_{j}$ is a $(3,3)$ extension of $\Lambda_{\Phi}$.

\begin{tabular}{||c|c|c|c||}
\hline \hline \multicolumn{4}{|c||}{$F_{4}$ : Order 3 (Partition 2) } \\
\hline$(0,0,4,0)$ & $(4,4,0,8)$ & $(4,4,4,8)$ & $(4,4,8,8)$ \\
$(0,0,8,0)$ & $(8,8,0,4)$ & $(8,8,8,4)$ & $(8,8,4,4)$ \\
\hline$(0,4,0,0)$ & $(4,0,4,4)$ & $(4,4,4,4)$ & $(4,8,4,4)$ \\
$(0,8,0,0)$ & $(8,0,8,8)$ & $(8,8,8,8)$ & $(8,4,8,8)$ \\
\hline$(0,4,0,4)$ & $(4,0,8,0)$ & $(4,4,8,4)$ & $(4,8,8,8)$ \\
$(0,8,0,8)$ & $(8,0,4,0)$ & $(8,8,4,8)$ & $(8,4,4,4)$ \\
\hline$(0,4,4,8)$ & $(4,0,8,8)$ & $(4,4,0,4)$ & $(4,8,4,0)$ \\
$(0,8,8,4)$ & $(8,0,4,4)$ & $(8,8,0,8)$ & $(8,4,8,0)$ \\
\hline \hline
\end{tabular}

Note that, for Lie algebras of type $A_{n}, B_{n}, C_{n}, D_{n}, F_{4}$ and $G_{2}$, the set $\mathfrak{M} / \Lambda_{\Phi}$ contains only elements of prime power order. However, the construction given in Lemma 6.1 shows that, if $L \cong E_{8}$, then $\mathfrak{M} / \Lambda_{\Phi}$ contains an element of order 6 . A complete description and count of the maximal functionals for all the isomorphism types of Lie algebras can be found in part II of this work, $[\mathrm{BP}$. We show, for example, in a noncomputational manner, that the orders of the elements of $\mathfrak{M} / \Lambda_{\Phi}$ are $1,2,3,4,5$ or 6 . In particular, the equality $e=f$ always holds, and there is a real reason for this to occur. Furthermore, some of the properties mentioned above, concerning the functionals for $G_{2}$ and $F_{4}$, are easily explained in this new context. The work in $[\mathrm{BP}]$ is based on techniques and results from the paper [D1], since we associate to each functional a certain semisimple subalgebra of $L$ of maximal rank.

Finally, Maple 9 worksheets, in text readable format, for specific computations in all the exceptional Lie algebras can be found on www .math.wisc.edu/ passman/ abstracts.html.

\section{LIE ALGEBRAS WITH TRIVIAL CENTERS}

If $L$ is one of the five exceptional simple finite-dimensional complex Lie algebras and if $\mathcal{F}=\left\{F_{i} \mid i \in \mathbb{Z}\right\}$ is a maximal bounded filtration of $L$, then we have not as yet been able to show that $F_{0}$ contains a Cartan subalgebra of $L$. In this section, we offer some general Lie theoretic lemmas which may eventually lead to a solution of this problem. As will be apparent, these results hold in a much more general context. To start with, let $L$ be an arbitrary Lie algebra over a field $K$. If $\mathfrak{Z}(L)$, the center of $L$, is nonzero, then $L$ has no maximal filtrations. Indeed, any bounded filtration can be properly enlarged by adjoining $\mathfrak{Z}(L)$ to each nonzero term and to any number of zero terms. Thus, in the following, we assume that $L$ is an arbitrary Lie algebra, but with the additional condition that $\mathfrak{Z}(L)=0$. The first result is fairly easy and not unexpected.

Lemma 7.1. Let $\mathcal{F}=\left\{F_{i} \mid i \in \mathbb{Z}\right\}$ be a maximal bounded filtration of $L$. If $G_{i}=\left\{x \in L \mid\left[x, F_{j}\right] \subseteq F_{i+j}\right.$ for all $\left.j \in \mathbb{Z}\right\}$, then $F_{i}=G_{i}$ for all $i$. 
Proof. It is clear that each $G_{i}$ is a subspace of $L$ and that $G_{i} \supseteq F_{i}$. Thus, it suffices to show that $\mathcal{G}=\left\{G_{i} \mid i \in \mathbb{Z}\right\}$ is a bounded filtration. To this end, note first that $\left[G_{i}, F_{k}\right] \subseteq F_{i+k}$ implies that

$$
\begin{aligned}
& {\left[\left[G_{i}, G_{j}\right], F_{k}\right] \subseteq\left[\left[G_{j}, F_{k}\right], G_{i}\right]+\left[\left[F_{k}, G_{i}\right], G_{j}\right] } \\
& \subseteq\left[F_{j+k}, G_{i}\right]+\left[F_{i+k}, G_{j}\right] \subseteq F_{i+j+k} .
\end{aligned}
$$

Thus $\left[G_{i}, G_{j}\right] \subseteq G_{i+j}$. Furthermore, $\left[G_{i}, F_{k}\right] \subseteq F_{i+k} \subseteq F_{i+1+k}$, so $G_{i} \subseteq G_{i+1}$ and $\mathcal{G}$ is indeed a filtration. Finally, if $F_{a}=0$ and $F_{b}=L$, then $G_{b}=L$ and $\left[G_{a-b}, L\right]=\left[G_{a-b}, F_{b}\right] \subseteq F_{a}=0$. In other words, $G_{a-b} \subseteq \mathfrak{Z}(L)$ and hence, by assumption, $G_{a-b}=0$. Thus $\mathcal{G}$ is bounded and the result follows.

In particular, with $i=0$, we have

$$
F_{0}=\left\{x \in L \mid\left[x, F_{j}\right] \subseteq F_{j} \text { for all } j \in \mathbb{Z}\right\}=\bigcap_{j} \mathfrak{N}_{L}\left(F_{j}\right),
$$

where $\mathfrak{N}_{L}$ denotes the normalizer in $L$. We will sharpen this result below.

To start with, if $x_{1}, x_{2}, \ldots, x_{n} \in L$, we use the symbol $\left[x_{1}, x_{2}, \ldots, x_{n}\right]_{\alpha}$ to denote any $n$-fold Lie product of these elements. Here, the subscript $\alpha$ is somehow presumed to hold the associativity information. One such product is obtained by associating to the left, and for this one, we drop the subscript. Thus

$$
\left[x_{1}, x_{2}, x_{3}, \ldots, x_{n}\right]=\left[\left[\left[\left[x_{1}, x_{2}\right], x_{3}\right], \ldots\right], x_{n}\right] .
$$

If $X_{1}, X_{2}, \ldots, X_{n}$ are $K$-subspaces of $L$, we use $\left[X_{1}, X_{2}, \ldots, X_{n}\right]_{\alpha}$ to denote the $K$-subspace of $L$ spanned by all $\left[x_{1}, x_{2}, \ldots, x_{n}\right]_{\alpha}$ with $x_{i} \in X_{i}$. The following is presumably well known.

Lemma 7.2. Let $X_{1}, X_{2}, \ldots, X_{n}$ be subspaces of $L$. Then, for all $\alpha$, we have

$$
\left[X_{1}, X_{2}, \ldots, X_{n}\right]_{\alpha} \subseteq \sum_{\sigma \in \operatorname{Sym}_{n}}\left[X_{\sigma(1)}, X_{\sigma(2)}, \ldots, X_{\sigma(n)}\right],
$$

where the right-hand summands all associate to the left.

Proof. We proceed by induction on $n$, the result being clear for $n=1$. If $n>1$, we can write $\left[X_{1}, X_{2}, \ldots, X_{n}\right]_{\alpha}=[R, S]$, where this indicates the last Lie product taken. Thus $R$ is an $r$-fold Lie product, $S$ is an $s$-fold Lie product and $r+s=n$. For fixed $n$, we proceed by induction on $\min (r, s)$ which, for convenience, we can assume to equal $s$. If $\min (r, s)=s=1$, then $S=X_{j}$ for some $j$, and say $j=n$. By induction, $R \subseteq \sum_{\sigma \in \operatorname{Sym}_{n-1}}\left[X_{\sigma(1)}, X_{\sigma(2)}, \ldots, X_{\sigma(n-1)}\right]$ and therefore

$$
\left[X_{1}, X_{2}, \ldots, X_{n}\right]_{\alpha}=\left[R, X_{n}\right] \subseteq \sum_{\sigma \in \operatorname{Sym}_{n-1}}\left[X_{\sigma(1)}, X_{\sigma(2)}, \ldots, X_{\sigma(n-1)}, X_{n}\right],
$$

and we are done.

On the other hand, if $\min (r, s)=s>1$, then $S=[U, V]$ with $U$ of length $u \geq 1$, $V$ of length $v \geq 1$, and with $u+v=s$. Now note that

$$
\left[X_{1}, X_{2}, \ldots, X_{n}\right]_{\alpha}=[R, S]=[R,[U, V]] \subseteq[[R, U], V]+[[R, V], U],
$$

and that each of $[[R, U], V]$ and $[[R, V], U]$ has length $n$, but with a smaller minimum than $[R, S]$. Thus, induction applies to each of these two summands, and the lemma is proved. 
As a first consequence, we obtain

Lemma 7.3. Let $\mathcal{F}=\left\{F_{i} \mid i \in \mathbb{Z}\right\}$ be a bounded filtration with $F_{a}=0$ for some $a<0$. Fix $\ell \geq 0$, let

$$
X=\left\{x \in L \mid\left[x, F_{j}\right] \subseteq F_{j+\ell} \text { for all } j<0\right\},
$$

and define $\operatorname{deg} X=\ell$. Furthermore, write $\operatorname{deg} F_{i}=i$ and if $C=\left[U_{1}, U_{2}, \ldots, U_{n}\right]_{\alpha}$ is an $n$-fold commutator with each $U_{k}$ either equal to $X$ or to some $F_{i}$, then we set $\operatorname{deg} C=\sum_{k=1}^{n} \operatorname{deg} U_{k}$. If $C \neq 0$, then $\operatorname{deg} C>a$.

Proof. We proceed by induction on $n \geq 1$. If $n=1$, then $C=F_{i}$ or $X$, and if $C=X$, then $\operatorname{deg} C=\operatorname{deg} X=\ell \geq 0>a$. On the other hand, if $C=F_{i}$ and $C \neq 0$, then surely $\operatorname{deg} C=i>a$.

Now suppose $n>1$. By Lemma 7.2

$$
0 \neq C=\left[U_{1}, U_{2}, \ldots, U_{n}\right]_{\alpha} \subseteq \sum_{\sigma \in \operatorname{Sym}_{n}}\left[U_{\sigma(1)}, U_{\sigma(2)}, \ldots, U_{\sigma(n)}\right],
$$

and hence at least one of the right-hand summands is nonzero. Thus, without loss of generality, we can assume that $C=\left[U_{1}, U_{2}, \ldots, U_{n}\right]$ is associated to the left, so that $C=\left[B, U_{n}\right]$, where $B=\left[U_{1}, U_{2}, \ldots, U_{n-1}\right]$. Obviously, $B \neq 0$ and, since $\operatorname{deg} C=\operatorname{deg} B+\operatorname{deg} U_{n}$, the result clearly follows if $\operatorname{deg} U_{n} \geq 0$. Thus, we need only consider the case where $U_{n}=F_{j}$ with $j<0$.

Since the adjoint map is a derivation, we have

$$
0 \neq C=\left[B, F_{j}\right] \subseteq \sum_{i=1}^{n-1}\left[U_{1}, \ldots, U_{i-1},\left[U_{i}, F_{j}\right], \ldots, U_{n-1}\right],
$$

and hence one of the right-hand summands is nonzero. Say it is the $i$ th term, so $0 \neq S_{i}=\left[U_{1}, \ldots, U_{i-1},\left[U_{i}, F_{j}\right], \ldots, U_{n-1}\right]$. If $U_{i}=F_{k}$, then $\left[U_{i}, F_{j}\right] \subseteq F_{k+j}$ and hence $0 \neq D=\left[U_{1}, \ldots, U_{i-1}, F_{j+k} \ldots, U_{n-1}\right]$. Thus, by induction, $\operatorname{deg} D>a$. But $\operatorname{deg} D=\operatorname{deg} C$, since $F_{k}$ and $F_{j}$ in $C$ are replaced by $F_{k+j}$ in $D$, so the result follows in this case.

On the other hand, if $U_{i}=X$, then $\left[U_{i}, F_{j}\right]=\left[X, F_{j}\right] \subseteq F_{j+\ell}$, since $j<0$, and hence $0 \neq E=\left[U_{1}, \ldots, U_{i-1}, F_{j+\ell}, \ldots, U_{n-1}\right]$. Thus, by induction, $\operatorname{deg} E>a$. But $\operatorname{deg} E=\operatorname{deg} C$, since $X$ and $F_{j}$ in $C$ are replaced by $F_{j+\ell}$ in $E$, and therefore the lemma is proved.

This now yields

Lemma 7.4. If $\mathcal{F}=\left\{F_{i} \mid i \in \mathbb{Z}\right\}$ is a maximal bounded filtration and $\ell \geq 0$ is a nonnegative integer, then

$$
F_{\ell}=\left\{x \in L \mid\left[x, F_{j}\right] \subseteq F_{j+\ell} \text { for all } j<0\right\} .
$$

In particular,

$$
F_{0}=\bigcap_{j<0} \mathfrak{N}_{L}\left(F_{j}\right) \supseteq \mathfrak{C}_{L}\left(F_{-1}\right),
$$

where $\mathfrak{C}_{L}\left(F_{-1}\right)$ is the centralizer of $F_{-1}$.

Proof. Set $X=\left\{x \in L \mid\left[x, F_{j}\right] \subseteq F_{j+\ell}\right.$ for all $\left.j<0\right\}$, so that $X$ is a subspace of $L$ containing $F_{\ell}$. Using the degree notation of the preceding lemma, we define

$$
G_{r}=\sum\left[U_{1}, U_{2}, \ldots, U_{n}\right]_{\alpha},
$$


where the sum is over all $n \geq 1$ and all choices of $\alpha$, with each $U_{k}$ either an $F_{i}$ or $X$, and with $\operatorname{deg}\left[U_{1}, U_{2}, \ldots, U_{n}\right]_{\alpha}=r$. It is clear that $\mathcal{G}=\left\{G_{r} \mid r \in \mathbb{Z}\right\}$ is a filtration containing $\mathcal{F}$, and $\mathcal{G}$ is bounded by Lemma 7.3. Thus, by maximality, we have $\mathcal{G}=\mathcal{F}$. In particular, $X=[X] \subseteq G_{\ell}=F_{\ell}$, and we have therefore obtained the reverse inclusion.

With this, we see that any maximal bounded filtration $\mathcal{F}=\left\{F_{i} \mid i \in \mathbb{Z}\right\}$ is determined by those $F_{j}$ with $j<0$. Surprisingly, we can also characterize the terms $F_{\ell}$ with $\ell<0$ in a similar manner to the above. However, here certain $F_{j}$ with $j \geq 0$ must also come into play.

Lemma 7.5. If $\mathcal{F}=\left\{F_{i} \mid i \in \mathbb{Z}\right\}$ is a maximal bounded filtration and $\ell<0$ is a negative integer, then

$$
F_{\ell}=\left\{x \in L \mid\left[x, F_{j}\right] \subseteq F_{j+\ell} \text { for all } j<-\ell\right\} .
$$

In particular, $\mathfrak{Z}\left(F_{-\ell-1}\right) \subseteq F_{\ell}$.

Proof. Set $X=\left\{x \in L \mid\left[x, F_{j}\right] \subseteq F_{j+\ell}\right.$ for all $\left.j<-\ell\right\}$, so that $X$ is a subspace of $L$ with $X \supseteq F_{\ell}$. We prove, by induction on $i \in \mathbb{Z}$, that $\left[X, F_{i}\right] \subseteq F_{i+\ell}$, and we are given that this inclusion holds for all $i<-\ell$.

Now suppose that $i \geq-\ell$ and that the inclusion holds for all smaller $i$. Since $i+\ell \geq 0$, we can use the characterization of $F_{i+\ell}$ given in the preceding lemma. To this end, let $j<0$ and note that

$$
\left[\left[X, F_{i}\right], F_{j}\right] \subseteq\left[\left[X, F_{j}\right], F_{i}\right]+\left[X,\left[F_{i}, F_{j}\right]\right] .
$$

By hypothesis, $\left[X, F_{j}\right] \subseteq F_{j+\ell}$, so $\left[\left[X, F_{j}\right], F_{i}\right] \subseteq\left[F_{j+\ell}, F_{i}\right] \subseteq F_{j+i+\ell}$. Furthermore, $\left[F_{i}, F_{j}\right] \subseteq F_{i+j}$ and, since $i+j<i$, induction yields $\left[X,\left[F_{i}, F_{j}\right]\right] \subseteq\left[X, F_{i+j}\right] \subseteq$ $F_{j+i+\ell}$. Thus, $\left[\left[X, F_{i}\right], F_{j}\right] \subseteq F_{j+i+\ell}$ for all $j<0$, so Lemma 7.4 implies that $\left[X, F_{i}\right] \subseteq F_{i+\ell}$, as required.

We now know that $\left[X, F_{i}\right] \subseteq F_{i+\ell}$ for all $i \in \mathbb{Z}$, and therefore Lemma 7.1 yields the result.

As we indicated, if $L$ has a maximal bounded filtration $\mathcal{F}=\left\{F_{i} \mid i \in \mathbb{Z}\right\}$, then $\mathfrak{Z}(L)=0$. It is possible that such maximal filtrations must necessarily satisfy $\mathfrak{Z}\left(F_{0}\right)=0$ in general. Here, we show at least

Lemma 7.6. If $L \neq 0$ and if $\mathcal{F}=\left\{F_{i} \mid i \in \mathbb{Z}\right\}$ is a maximal bounded filtration, then $F_{0}$ is not abelian.

Proof. Suppose, by way of contradiction, that $F_{0}$ is abelian. By the previous lemma, with $\ell=-1$, we see that $F_{0}=\mathfrak{Z}\left(F_{0}\right) \subseteq F_{-1}$, so $F_{-1}=F_{0}$. Furthermore, since $F_{0} \supseteq \mathfrak{C}\left(F_{-1}\right)=\mathfrak{C}\left(F_{0}\right)$, by Lemma 7.4, and since $L \neq 0$, it follows that $F_{0} \neq 0$. Now suppose that $F_{0}=F_{-1}=\cdots=F_{\ell+1}$ for some integer $\ell \leq-2$. We apply the previous lemma to show that $F_{0} \subseteq F_{\ell}$.

To start with, since $F_{0}$ is abelian, if $j \leq 0$, then $\left[F_{0}, F_{j}\right]=0 \subseteq F_{j+\ell}$. On the other hand, if $0<j<-\ell$, then $0>-j>\ell$ and $0>j+\ell>\ell$. Thus $\left[F_{0}, F_{j}\right]=\left[F_{-j}, F_{j}\right] \subseteq F_{0}=F_{j+\ell}$. It now follows from Lemma 7.5 that $F_{0} \subseteq F_{\ell}$ and hence that $F_{0}=F_{\ell}$. Continuing in this manner, we see that $F_{0}=F_{k}$ for all $k \leq 0$, and this contradicts the fact that $\mathcal{F}$ is bounded.

Hopefully, these lemmas will contribute to a later work on this subject, yielding stronger and more interesting results of this type. 


\section{Filtrations OVER ARChimedeAN ORDERED GROUPS}

Let $L$ be a Lie algebra over the field $K$ and let $\mathbb{G}$ be an ordered group, written additively. Then $\mathcal{F}=\left\{F_{i} \mid i \in \mathbb{G}\right\}$ is a $\mathbb{G}$-filtration of $L$ if each $F_{i}$ is a $K$-subspace of $L,\left[F_{i}, F_{j}\right] \subseteq F_{i+j}$ for all $i, j \in \mathbb{G}$, and $F_{i} \subseteq F_{j}$ whenever $i \leq j$. Of course, one also assumes that $\bigcup_{i} F_{i}=L$ and $\bigcap_{i} F_{i}=0$. In particular, $F_{0}$ is a Lie subalgebra of $L$, and each $F_{i}$ is an ad $F_{0}$-submodule. Again, we say that $\mathcal{F}$ is bounded if there exist $\ell, \ell^{\prime} \in \mathbb{G}$ with $F_{\ell}=0$ and $F_{\ell^{\prime}}=L$.

As in the case when $\mathbb{G}=\mathbb{Z}$, we would like the boundedness of $\mathcal{F}$ to imply that each $F_{i}$ with $i<0$ is ad-nilpotent on $L$. This would, of course, follow if for any $i<0$ there exists a positive integer $m$ with $m i+\ell^{\prime} \leq \ell$. Thus, if we wish to restrict our study to bounded filtrations that enjoy properties similar to those of $\mathbb{Z}$-filtrations, it makes sense to assume that $\mathbb{G}$ is an Archimedean ordered group. In this case, one knows from $[\mathrm{Ho}$ ] that $\mathbb{G}$ is an abelian group, and then from $[\mathrm{Ba}]$ that $\mathbb{G}$ is an additive subgroup of the reals $\mathbb{R}$. In particular, either $\mathbb{G} \cong \mathbb{Z}$ or $\mathbb{G}$ is a dense subgroup of $\mathbb{R}$. Of course, in view of the skew symmetry of the Lie product, it certainly makes sense to assume that $\mathbb{G}$ is abelian for any Lie filtration.

Since the $\mathbb{G} \cong \mathbb{Z}$ case has already been considered, we will restrict our attention in this section to dense subgroups of $\mathbb{R}$. Furthermore, since the arguments for dense subgroups are quite similar, and sometimes easier, than those for $\mathbb{Z}$, we will merely stress the differences when they occur, and just sketch the proofs. For convenience, we say that the filtration $\mathcal{F}=\left\{F_{i} \mid i \in \mathbb{G}\right\}$ is upper continuous if the condition $F_{i}=\bigcap_{i^{\prime}>i} F_{i^{\prime}}$ holds for all $i \in \mathbb{G}$.

Lemma 8.1. Let $L$ be a Lie algebra and let $\mathbb{G}$ be a dense subgroup of the additive group of real numbers $\mathbb{R}$. If $\mathcal{F}=\left\{F_{i} \mid i \in \mathbb{G}\right\}$ is a bounded filtration of $L$, then $\mathcal{F}$ is contained in a bounded upper continuous filtration $\overline{\mathcal{F}}$. In particular, if $\mathcal{F}$ is maximal, then it must be upper continuous.

Proof. Let $\overline{\mathcal{F}}=\left\{\bar{F}_{i} \mid i \in \mathbb{G}\right\}$ be defined by $\bar{F}_{i}=\bigcap_{i^{\prime}>i} F_{i}$. If $i, j, k \in \mathbb{G}$ with $k>i+j$, then the denseness of $\mathbb{G}$ implies that there exist $i^{\prime}>i$ and $j^{\prime}>j$ with $i+j<i^{\prime}+j^{\prime} \leq k$. With this, it follows that $\left[\bar{F}_{i}, \bar{F}_{j}\right] \subseteq \bar{F}_{i+j}$, and then $\overline{\mathcal{F}}$ is easily seen to be a bounded filtration of the Lie algebra $L$ containing $\mathcal{F}$.

Next, suppose that $L=A \oplus B$ is a direct sum of the two Lie algebras $A$ and $B$. If $\mathcal{A}=\left\{A_{i} \mid i \in \mathbb{G}\right\}$ and $\mathcal{B}=\left\{B_{i} \mid i \in \mathbb{G}\right\}$ are bounded filtrations of $A$ and $B$, respectively, and if, as before, we define $L_{i}=A_{i} \oplus B_{i} \subseteq L$, then $\mathcal{L}=\left\{L_{i} \mid i \in \mathbb{G}\right\}$ is easily seen to be a bounded $\mathbb{G}$-filtration of $L$. Again, we write $\mathcal{L}=\mathcal{A} \oplus \mathcal{B}$ and say that $\mathcal{L}$ is the sum of $\mathcal{A}$ and $\mathcal{B}$. With this notation, the proof of Lemma 1.4 immediately yields

Lemma 8.2. Let $L$ be a $K$-Lie algebra and let $\mathcal{F}=\left\{F_{i} \mid i \in \mathbb{G}\right\}$ be a bounded $\mathbb{G}$-filtration of $L$.

(i) Suppose $L=A \oplus B$ is a direct sum of the $K$-Lie algebras $A$ and $B$. Then $\mathcal{F}$ is maximal if and only if $\mathcal{F}=\mathcal{A} \oplus \mathcal{B}$ with $\mathcal{A}$ and $\mathcal{B}$ maximal bounded $\mathbb{G}$-filtrations of $A$ and $B$, respectively.

(ii) Suppose $L$ is a finite-dimensional complex semisimple Lie algebra, and write $L=L_{1} \oplus L_{2} \oplus \cdots \oplus L_{k}$ as a finite direct sum of simple Lie algebras. Then $\mathcal{F}$ is maximal if and only if it is a sum $\mathcal{F}=\mathcal{F}_{1} \oplus \mathcal{F}_{2} \oplus \cdots \oplus F_{k}$, where each $\mathcal{F}_{j}$ is a maximal bounded $\mathbb{G}$-filtration of $L_{j}$. 
Thus, the study of maximal bounded $\mathbb{G}$-filtrations in semisimple complex Lie algebras also reduces to the simple case, and then it is clear that the work of Section 2 carries over to this context with just minor changes. To start with, we must replace $F_{-1}$ by the Lie subalgebra $F_{0^{-}}=\bigcup_{j<0} F_{j}$ in the proof of Lemma 2.2. Furthermore, we must cite the references $[\mathrm{P}$, Theorem 6.5 and Lemma 6.7] rather than $[\mathrm{P}$, Lemmas 2.5 and 5.7] in the proofs of Propositions 2.5 and 2.6. With these modifications, we obtain the following $\mathbb{G}$-analogs of the latter two propositions.

Proposition 8.3. Let $K$ be a field, let $L=\mathfrak{s l}_{m}(K)$ with $m \geq 2$, and suppose that $\mathcal{F}=\left\{F_{i} \mid i \in \mathbb{G}\right\}$ is a maximal bounded $\mathbb{G}$-filtration of $L$. If $m \neq 0$ in $K$, then $F_{0}$ contains a Cartan subalgebra of $L$.

Proposition 8.4. Let $K$ be an algebraically closed field of characteristic $\neq 2$, let * be an involution of $\mathrm{M}_{m}(K)$ of the first kind, and let $L$ be the Lie subalgebra of $\mathfrak{g l}_{m}(K)$ consisting of all the skew-symmetric matrices under the action of $*$. If $\mathcal{F}=\left\{F_{i} \mid i \in \mathbb{G}\right\}$ is a maximal bounded $\mathbb{G}$-filtration of $L$ and if $m \geq 3$, then $F_{0}$ contains a Cartan subalgebra of $L$.

These, of course, now combine to yield

Corollary 8.5. Let $K$ be the field of complex numbers and let $L$ be a simple $K$-Lie algebra of type $A_{n}, B_{n}, C_{n}$ or $D_{n}$. If $\mathcal{F}=\left\{F_{i} \mid i \in \mathbb{G}\right\}$ is a maximal bounded $\mathbb{G}$-filtration of $L$, then $F_{0}$ contains a Cartan subalgebra of $L$.

Let $L$ be an arbitrary finite-dimensional simple Lie algebra over the complex numbers $K$. We say that a bounded $\mathbb{G}$-filtration $\mathcal{F}=\left\{F_{i} \mid i \in \mathbb{G}\right\}$ of $L$ is Cartan if $F_{0}$ contains a Cartan subalgebra $L_{0}$ of $L$. In view of the preceding corollary, our next goal is obviously to determine the maximal bounded $\mathbb{G}$-filtrations of $L$ that are also Cartan. Note that, if $\mathcal{G}=\left\{G_{i} \mid i \in \mathbb{G}\right\}$ is a filtration containing the filtration $\mathcal{F}$, then $G_{0} \supseteq F_{0} \supseteq L_{0}$ and hence $\mathcal{G}$ is also Cartan. Let $L=L_{0}+\sum_{\alpha \in \Phi} L_{\alpha}$ be the root space decomposition of $L$ with respect to $L_{0}$. Here $\Phi=\Phi(L)$ is the set of roots of $L$, and each $L_{\alpha}$ with $\alpha \in \Phi$ is a 1-dimensional subspace of $L$. As before, our basic tool here is the degree function $f$ of $\mathcal{F}$, this time defined by

$$
f(\alpha)=\inf \left\{i \in \mathbb{G} \mid L_{\alpha} \subseteq F_{i}\right\} .
$$

Since $\mathcal{F}$ is bounded, $f(\alpha)$ is a well-defined real number for all $\alpha \in \Phi$. Again, we write $\Phi^{\prime}=\Phi \cup 0$ and define $f(0)=0$.

Lemma 8.6. Let $\mathcal{F}=\left\{F_{i} \mid i \in \mathbb{G}\right\}$ be a Cartan filtration of the simple Lie algebra $L$ and let $f$ be its degree function. Assume that $\mathcal{F}$ is upper continuous.

(i) For each $i \in \mathbb{G}$, we have $F_{i}=\sum L_{\alpha}$, where the sum is over all $\alpha \in \Phi^{\prime}$ with $f(\alpha) \leq i$. In particular, $f$ uniquely determines the filtration.

(ii) If $\alpha, \beta$ and $\alpha+\beta$ are contained in $\Phi$, then $f(\alpha)+f(\beta) \geq f(\alpha+\beta)$. Furthermore, $f(\alpha)+f(-\alpha) \geq 0$.

(iii) If $\mathcal{G}=\left\{G_{i} \mid i \in \mathbb{G}\right\}$ is a second upper continuous Cartan filtration of $L$ with degree function $g$, then $\mathcal{G}$ contains $\mathcal{F}$ if and only if $g(\alpha) \leq f(\alpha)$ for all roots $\alpha \in \Phi$.

Proof. We begin with part (i). Since $F_{0} \supseteq L_{0}$ and since each $F_{i}$ is an ad $F_{0}$-module, it follows that each $F_{i}$ is a direct sum of suitable $L_{\alpha}$ s along with $F_{i} \cap L_{0}$. As in the proof of Lemma 3.1(i), we know that $L_{0} \cap F_{i}=0$ if $i<0$, and $L_{0} \cap F_{i}=L_{0}$ if $i \geq 0$. Thus, since $\lambda(0)=0$, this term behaves correctly. Furthermore, by the 
definition of the function $f$, it is clear that $L_{\alpha}$ does not occur in $F_{i}$ if $i<f(\alpha)$. On the other hand, suppose $i \geq f(\alpha)$ and let $i^{\prime} \in \mathbb{G}$ with $i^{\prime}>i \geq f(\alpha)$. Then again, the definition of $f$ implies that $L_{\alpha} \subseteq F_{i^{\prime}}$, and upper continuity yields the result. Parts (ii) and (iii) follow as in Lemma 3.1(ii), (iii).

Now let $V$ be the real inner product space determined by $\Phi(L)$, the root set of $L$. As before, if $\lambda: V \rightarrow \mathbb{R}$ is a linear functional, we define the dual filtration $\mathcal{F}_{\lambda}=\left\{F_{i} \mid i \in \mathbb{G}\right\}$ by $F_{i}=\sum_{\alpha} L_{\alpha}$, where the sum is over all $\alpha \in \Phi^{\prime}$ with $\lambda(\alpha) \leq i$.

Lemma 8.7. If $\lambda: V \rightarrow \mathbb{R}$, then $\mathcal{F}_{\lambda}$ is an upper continuous Cartan filtration of $L$ with degree function given by $f_{\lambda}(\alpha)=\lambda(\alpha)$. Furthermore, $\mathcal{F}_{\lambda} \subseteq \mathcal{F}_{\mu}$ if and only if the functionals $\lambda$ and $\mu$ are equal.

Proof. The first part is clear. For the second, we know that $F_{\lambda} \subseteq F_{\mu}$ if and only if $\mu(\alpha)=f_{\mu}(\alpha) \leq f_{\lambda}(\alpha)=\lambda(\alpha)$ for all roots $\alpha$. But $-\alpha$ is also a root, so $-\mu(\alpha)=$ $\mu(-\alpha) \leq \lambda(-\alpha)=-\lambda(\alpha)$, and we obtain the reverse inequality $\lambda(\alpha) \leq \mu(\alpha)$. Thus $\mathcal{F}_{\lambda} \subseteq \mathcal{F}_{\mu}$ implies that $\lambda=\mu$, as required.

This is, of course, markedly different from the case of $\mathbb{Z}$-filtrations where the ceiling function \lceil\rceil brings number theoretic considerations into play. The importance of these dual filtrations is again based on

Lemma 8.8. Let $\mathcal{F}=\left\{F_{i} \mid i \in \mathbb{G}\right\}$ be a Cartan filtration of the simple Lie algebra $L$. If $V$ is the root space of $L$, then there exists a linear functional $\lambda: V \rightarrow \mathbb{R}$ such that $\mathcal{F} \subseteq \mathcal{F}_{\lambda}$.

Proof. In view of Lemma 8.1, we can assume that $\mathcal{F}$ is upper continuous. In particular, $\mathcal{F}$ is determined by its degree function $f$ and, since the proof of Lemma 3.6 only involves degree functions, it clearly carries over to this context.

It is an easy consequence of the preceding two lemmas that each $\mathcal{F}_{\lambda}$ is a maximal $\mathbb{G}$-filtration, and with this, our main result on filtrations over dense Archimedean ordered groups follows immediately. Indeed, we have

Theorem 8.9. Let $L$ be a finite-dimensional complex simple Lie algebra, let $\mathbb{G}$ be a dense subgroup of $\mathbb{R}$, and let $\mathcal{F}=\left\{F_{i} \mid i \in \mathbb{G}\right\}$ be a bounded $\mathbb{G}$-filtration. Suppose $H$ is a Cartan subalgebra of $L$, write $V$ for the root space of $L$, and let $\widehat{V}=\operatorname{Hom}(V, \mathbb{R})$ denote its dual space.

(i) If $\mathcal{F}$ is maximal and if $L$ is of type $A_{n}, B_{n}, C_{n}$ or $D_{n}$, then $F_{0}$ contains a Cartan subalgebra of $L$.

(ii) The maximal filtrations $\mathcal{F}$ with $F_{0} \supseteq H$ are precisely the filtrations $\mathcal{F}_{\lambda}$ with $\lambda$ a linear functional of $V$. In particular, there is a one-to-one correspondence between these maximal filtrations and the elements of $\widehat{V}$.

Again, we suspect that part (i) above is also true for the exceptional Lie algebras $E_{6}, E_{7}, E_{8}, F_{4}$ and $G_{2}$.

\section{REFERENCES}

[Ba] R. Baer, Zur Topologie der Gruppen, J. Reine Angew. Math. 160 (1929), 208-226.

[B] Y. Barnea, Maximal graded subalgebras of loop toroidal Lie algebras, Algebr. Represent. Theory. 8 (2005), no. 2, 165-171. MR2162280

[BSZ] Y. Barnea, A. Shalev and E.I. Zelmanov, Graded subalgebras of affine Kac-Moody algebras, Israel J. Math. 104 (1998), 321-334. MR1622319 (99d:17025)

[BP] Y. Barnea and D. S. Passman, Filtrations in semisimple Lie algebras, II, to appear. 
[Bo] N. Bourbaki, Lie Groups and Lie Algebras: Chapters 4-6, Springer-Verlag, Berlin, 2002. MR 1890629 (2003a:17001)

[D1] E. B. Dynkin, Semisimple subalgebras of semisimple Lie algebras, AMS Translations (2) 6 (1957), 111-244.

[D2] E. B. Dynkin, Maximal subgroups of the classical groups, AMS Translations (2) 6 (1957), 245-378. MR0049903 (14:244d)

[He] I. N. Herstein, Rings with Involution, Univ. Chicago Press, Chicago, 1976. MR0442017 $(56: 406)$

[Ho] O. Hölder, Die Axiome der Quantität und die Lehre vom Mass, Ber. Verh. Sächs. Ges. Wiss. Leipzig. Math.-Phys. Kl. 53 (1901), 1-64.

[Hu] J. E. Humphreys, Introduction to Lie Algebras and Representation Theory, second printing, Springer-Verlag, New York, 1972. MR0323842 (48:2197)

[J] N. Jacobson, Lie Algebras, Wiley-Interscience, New York, 1962. MR0143793 (26:1345)

[K] V. G. Kac, Infinite Dimensional Lie Algebras, Cambridge Univ. Press, Cambridge, 1990. MR:1104219 (92k:17038)

[P] D. S. Passman, Filtrations in semisimple rings, Trans. AMS 357 (2005), no. 12, 5051-5066. MR2165397

Department of Mathematics, Royal Holloway, University of London, Egham, Surrey TW20 0EX, UNITED KINGDOM

E-mail address: y.barnea@rhul.ac.uk

Department of Mathematics, University of Wisconsin-Madison, Madison, Wisconsin 53706

E-mail address: passman@math.wisc.edu 